\title{
Structural analysis of massive galaxies using HST deep imaging at $z<0.5$
}

\author{
Sandra N. dos Reis ${ }^{1,2}$, Fernando Buitrago ${ }^{1,2}$, Polychronis Papaderos ${ }^{1,3}$, Israel Matute ${ }^{1}$, José Afonso ${ }^{1,2}$, \\ Stergios Amarantidis ${ }^{1,2}$, Iris Breda ${ }^{3}$, Jean M. Gomes ${ }^{3}$, Andrew Humphrey ${ }^{3}$, Catarina Lobo ${ }^{3,4}$, Silvio Lorenzoni ${ }^{1}$, \\ Cirino Pappalardo ${ }^{1}$, Ana Paulino-Afonso ${ }^{1}$, and Tom Scott ${ }^{3}$ \\ 1 Instituto de Astrofísica e Ciências do Espaço, Universidade de Lisboa, OAL, Tapada da Ajuda, 1349-018 Lisboa, Portugal \\ 2 Departamento de Física, Faculdade de Ciências da Universidade de Lisboa, Edifício C8, Campo Grande, 1749-016 Lisboa, Portugal \\ e-mail: sreis@oal.ul.pt \\ 3 Instituto de Astrofísica e Ciências do Espaço, Universidade do Porto, CAUP, Rua das Estrelas, 4150-762 Porto, Portugal \\ ${ }^{4}$ Departamento de Física e Astronomia, Faculdade de Ciências, Universidade do Porto, Rua do Campo Alegre 687, 4169-007 Porto, \\ Portugal
}

Received 9 July 2019 / Accepted 26 November 2019

\begin{abstract}
Context. The most massive galaxies $\left(M_{\text {stellar }} \geq 10^{11} M_{\odot}\right)$ in the local Universe are characterized by a bulge-dominated morphology and old stellar populations, in addition to being confined to a tight mass-size relation. Identifying their main components can provide insights into their formation mechanisms and subsequent mass assembly.

Aims. Taking advantage of Hubble Space Telescope (HST) CANDELS data, we analyze the lowest redshift $(z<0.5)$ massive galaxies in the $H$ and $I$ band in order to disentangle their structural constituents and study possible faint non-axisymmetric features.

Methods. Our final sample consists of 17 massive galaxies. Due to the excellent HST spatial resolution for intermediate redshift objects, they are hard to model by purely automatic parametric fitting algorithms. We performed careful single and double (bulge-disk decompositions) Sérsic fits to their galaxy surface brightness profiles. We compare the model color profiles with the observed ones and also derive multi-component global effective radii attempting to obtain a better interpretation of the mass-size relation. Additionally, we test the robustness of our measured structural parameters via simulations.

Results. We find that the Sérsic index does not offer a good proxy for the visual morphological type for our sample of massive galaxies. Our derived multi-component effective radii give a better description of the size of our sample galaxies than those inferred from single Sérsic models with GALFIT. Our galaxy population lies on the scatter of the local mass-size relation, indicating that these massive galaxies have not experienced a significant growth in size since $z \sim 0.5$. Interestingly, the few outliers are late-type galaxies, indicating that spheroids must reach the local mass-size relation earlier. For most of our sample galaxies, both single- and multi-component Sérsic models with GALFIT show substantial systematic deviations from the observed surface brightness profiles in the outskirts. These residuals may be partly due to several factors, namely a nonoptimal data reduction for low surface brightness features or the existence of prominent stellar haloes for massive galaxies, or they could also arise from conceptual shortcomings of parametric $2 \mathrm{D}$ image decomposition tools. They consequently propagate into galaxy color profiles. This is a significant obstacle to the exploration of the structural evolution of galaxies, which calls for a critical assessment and refinement of existing surface photometry techniques.
\end{abstract}

Key words. galaxies: evolution - galaxies: structure - galaxies: photometry

\section{Introduction}

The most massive $\left(M_{\text {stellar }} \geq 10^{11} M_{\odot}\right)$ galaxies in the Universe appear to have undergone a dramatic transformation in their structural properties across cosmic time, from compact star-forming disks to huge red and dead spheroidal galaxies (e.g., Trujillo et al. 2007; Buitrago et al. 2008, 2013; van Dokkum et al. 2010; Huertas-Company et al. 2015). However, how galaxies acquire their mass and how they evolve morphologically are still open questions. Therefore, the study of their structural properties is indispensable for a thorough understanding of the formation and evolution of these galaxies.

Currently, the most favored galaxy formation model for massive galaxies involves a two-phase build-up scenario. This scenario predicts a rapid formation phase at $2<z<6$ dominated by in situ star formation (Oser et al. 2010), and a subsequent phase of stellar mass growth through multiple minor mergers (e.g., López-Sanjuan et al. 2010, 2011, 2012; Bluck et al. 2012; Mármol-Queraltó et al. 2012; Ferreras et al. 2014, 2017) that may transform them into present-day spheroids. Following this scenario, Hopkins et al. (2009) studied the radial surface density profiles of high- $(z>2)$ and low-redshift $(z \sim 0)$ massive galaxies, comparing directly the observed profiles at the same physical radii. They demonstrate that the central components of local massive spheroids are not different from the high-redshift systems. Inside the same physical radii, the stellar surface mass densities of many of the local ellipticals are comparable to those of high-redshift objects, differing in effective radius $\left(r_{\mathrm{e}}\right)$ mainly due to an extended low surface brightness envelope in the lowredshift spheroids, as opposed to the steep fall of profiles of highredshift objects. A similar study from Bezanson et al. (2009) strengthens this conclusion: the central surface densities of the 
high-redshift systems are comparable with the average densities within $1 \mathrm{kpc}$ of the low-redshift ellipticals, implying an inside-out growth scenario whereby the compact high-redshift $(z \geq 2)$ galaxy remnants are contained within the cores of nearby early-type galaxies. To date, however, there have been no direct detections of compact old cores within elliptical galaxies, and the main drivers of the dramatic morphological and structural evolution of massive galaxies across redshift remain unclear. A subsequent study by de la Rosa et al. (2016) makes use of catalogs based on the Sloan Digital Sky Survey (SDSS) with bulge plus disk $(B+D)$ decompositions without restricting the morphology of the host galaxies. This study tests the hypothesis that the massive compact high-redshift galaxies were already quiescent in terms of star formation (termed "red nuggets" in Damjanov et al. 2009) and are hidden in the cores of presentday galaxies. In that study the central regions of both spheroids and disks are treated as independent entities, and are evaluated according to their compactness. The authors show that the cores of local massive galaxies are structurally equivalent to red nuggets at $z \sim 1.5$.

Studying the structural components of massive galaxies can yield a better understanding of the different processes behind the assembly of this galaxy population. Several works on photometric decomposition of galaxies contributed to the discovery of many scaling relations (e.g., Faber \& Jackson 1976; Kormendy 1977; Djorgovski \& Davis 1987), and particularly for the case of massive galaxies, it has been established that they closely follow the mass-size relation in the local Universe (e.g., Shen et al. 2003). One should bear in mind, however, that while single Sérsic profiles are frequently used as a standard model to automatically fit large samples (e.g., Simard et al. 2002; van der Wel et al. 2012; Griffith et al. 2012), galaxies are more complex and their structural characterization typically requires several components. Traditionally, massive galaxies were described as the outcome of the superposition of a central spheroidal bulge described by the de Vaucouleurs (1948) law and a more extended exponential disk (Freeman 1970). However, the increasing quality and lower limiting surface brightness $(\mu)$ of imaging data has brought to light new features within the bulges, such as faint spiral-like patterns (e.g., Kehrig et al. 2012; Gomes et al. 2016); surface brightness profiles (SBPs) with a Sérsic index $n \simeq 1$ to 2 , generally attributed to a pseudo-bulge (see, e.g., Kormendy \& Kennicutt 2004); or a significant fraction of stellar mass located within the haloes of massive ETGs that has likely accumulated through multiple minor merging events (Kaviraj et al. 2015; Buitrago et al. 2017).

A direct consequence of the findings referred to above is the necessity of moving from integrated properties to resolved quantities within galaxies, requiring two-component fitting as a way to provide a deeper insight into the different processes in place to assemble the main constituents of galaxies. Although there are already many computationally expensive works involving $\mathrm{B}+\mathrm{D}$ decomposition of large samples at low redshift (e.g., Allen et al. 2006; Simard et al. 2011; Lackner \& Gunn 2012; Mendel et al. 2014; Meert et al. 2015; Lange et al. 2016; Dimauro et al. 2018, to mention but a few), a critical amount of post-processing is required to evaluate the quality of the fits. However, this task requires a large amount of interactive work for the inspection of the fitting results to assure the physical solutions of the models. While such a detailed inspection of the modeling output is both feasible and mandatory for individual galaxies, it is generally out of reach in the case of quasi-automated studies of large galaxy samples.

The present work is dedicated to the structural analysis of 17 low-redshift $(z<0.5$, to avoid the issue of cosmological dimming, which increases strongly with redshift) massive galaxies from CANDELS using multi-band surface photometry and $\mathrm{B}+\mathrm{D}$ profile decomposition. The availability of data from the Hubble Space Telescope (HST) permits a superb investigation (in terms of spatial resolution) of the galaxy structure of our target galaxies, while the Cosmic Assembly Near-infrared Deep Extragalactic Legacy Survey (CANDELS ${ }^{1}$, Grogin et al. 2011; Koekemoer et al. 2011) "Deep" segment of the survey allows us to obtain limiting magnitudes that were previously unreachable. These HST images at low- $z(z<0.5)$ therefore provide us with a sample of relatively well-resolved galaxies from which we can extract precious information, especially in the low surface brightness regime.

In Sect. 2 we describe the data and sample selection. We specify the methodology used for the profile fitting, which takes advantage of state-of-the-art source extraction and surface photometry packages, in Sect. 3. We present and discuss the various derived structural properties of the galaxy sample (e.g., Sérsic indices, bulge-to-total luminosity ratios) and the analysis with respect to integral properties (integrated magnitude and total stellar mass) in Sect. 4. A summary and our conclusions follow in Sect. 5. Simulations performed to quantify the accuracy of our structural parameters measures are included in Appendix A.

Throughout the paper we adopt a cosmology with $\Omega_{\mathrm{m}}=0.3$, $\Omega_{\Lambda}=0.7$, and $H_{0}=70 \mathrm{~km} \mathrm{~s}^{-1} \mathrm{Mpc}^{-1}$. Magnitudes are provided in the AB system (Oke \& Gunn 1983).

\section{Data and sample selection}

The HST imaging data were retrieved from the CANDELS survey (Grogin et al. 2011; Koekemoer et al. 2011), while our sample was selected from the 3D-HST catalog (Skelton et al. 2014), since this catalog includes stellar masses and spectroscopic redshifts for the galaxies within all five CANDELS fields. CANDELS covers a total of $\sim 800$ square arcminutes widely distributed, and contains a Deep and a Wide component. The Deep portion of the survey comprises about $125 \mathrm{arcmin}^{2}$ to $\sim 10$-orbit depth within the GOODSNorth and GOODS-South fields. The remaining area includes the shallower Wide component, distributed over all five fields, to $\sim 2-$ HST orbit depth. CANDELS is a survey optimized for the detection of galaxies at high redshifts, and as it is very deep and wide, it allows us to retrieve nearby massive galaxies with a very high signal-to-noise ratio $(\mathrm{S} / \mathrm{N})$.

For our analysis we used two filters from CANDELS: the $F 160 W$ filter (WFC3 $H$ band) with a scale of $0.06^{\prime \prime} /$ pixel and pivot wavelength equal to $1536.9 \mathrm{~nm}$ corresponding to the reddest HST filter (the most representative of the total stellar component), and the $F 814 W$ filter (ACS $I$ band) which has a scale of $0.03^{\prime \prime} / \mathrm{pixel}$ and pivot wavelength of $805.7 \mathrm{~nm}$ and is the most representative of the optical rest frame in the redshift range considered. From a total of 207967 sources in all five CANDELS/3D-HST fields, and with the simple selection criteria of stellar masses higher than $10^{11} M_{\odot}$ and (spectroscopic, when available) redshifts $\leq 0.5$, we extracted 68 sources. However, since most of these sources are stars or objects not observed with the WFC3 F160W filter, the final sample consists of 17 massive galaxies (one object not observed in the ACS $I$ band). The upper limit for the redshift was chosen to assure that we obtain the galaxies with an adequate $\mathrm{S} / \mathrm{N}$. All objects in our sample are located in the Wide imaging of CANDELS, reaching $3 \sigma$ limiting magnitudes for extended sources of $H_{F 160 W} \sim 28.3 \mathrm{mag} \mathrm{arcsec}^{2}$ in

\footnotetext{
http://arcoiris.ucolick.org/candels/data_access/
} Latest_Release.html 
Table 1. Summary of the properties for our sample of galaxies, taken from the 3D-HST catalog (Skelton et al. 2014).

\begin{tabular}{lcccccc}
\hline \hline 3D-HST ID & Field & $\begin{array}{c}\text { RA } \\
{[\mathrm{J} 2000]}\end{array}$ & $\begin{array}{c}\text { Dec } \\
{[\mathrm{J} 2000]}\end{array}$ & $z$ & $\begin{array}{c}\log M_{\text {stellar }} \\
{\left[M_{\odot}\right]}\end{array}$ & Visual type \\
\hline 37194 & AEGIS & 214.7928 & 52.8643 & 0.0820 & 11.02 & LTGb \\
30654 & GOODS-N & 189.4151 & 62.2974 & 0.2133 & 11.10 & LTG \\
37587 & GOODS-N & 189.3515 & 62.3660 & 0.2810 & 11.29 & LTG \\
19195 & COSMOS & 150.0581 & 2.38042 & 0.3474 & 11.03 & ETG \\
3740 & AEGIS & 215.1804 & 52.9960 & 0.3510 & 11.02 & ETG \\
20050 & COSMOS & 150.0806 & 2.39026 & 0.3531 & 11.05 & ETG \\
10876 & COSMOS & 150.0951 & 2.30050 & 0.3604 & 11.02 & ETG \\
21796 & GOODS-N & 189.4206 & 62.2548 & 0.3769 & 11.22 & LTG \\
18935 & AEGIS & 215.2186 & 53.0791 & $0.39^{(*)}$ & 11.10 & LTG \\
7013 & GOODS-N & 188.9807 & 62.1809 & 0.4090 & 11.27 & LTG \\
21604 & UDS & 34.55835 & -5.2059 & $0.42^{(*)}$ & 11.11 & LTG \\
25781 & COSMOS & 150.1822 & 2.45136 & 0.4212 & 11.01 & ETG \\
1996 & COSMOS & 150.0907 & 2.20566 & 0.4269 & 11.12 & ETG \\
21306 & GOODS-N & 189.4720 & 62.2483 & $0.47^{(*)}$ & 11.06 & ETG \\
23956 & GOODS-N & 189.0092 & 62.2638 & $0.47^{(*)}$ & 11.87 & LTG \\
13942 & COSMOS & 150.1893 & 2.32564 & 0.4825 & 11.05 & LTGb \\
4735 & UDS & 34.43567 & -5.2562 & 0.4940 & 11.17 & ETG \\
\hline
\end{tabular}

Notes. Each column shows, from left to right: (1) galaxy ID matching the identifiers from the 3D-HST master catalog, (2) CANDELS field, (3) Right Ascension (deg), (4) Declination (deg), (5) redshift, (6) stellar mass in units of $\log \left(M_{\odot}\right)$, (7) visual morphology represented as early-type (ETG), late-type (LTG), and late-type with a bar (LTGb). ${ }^{(*)}$ Photometric redshifts.

the COSMOS field, $H_{F 160 W} \sim 28.5$ mag $\operatorname{arcsec}^{2}$ in the GOODS-N field, and $H_{F 160 W} \sim 28.6 \mathrm{mag}$ arcsec ${ }^{2}$ in the UDS and EGS fields.

By applying a visual morphological classification, our final sample contains nine late-type galaxies (LTGs; disk-dominated, two of them with a bar) and eight early-type galaxies (ETGs; spheroid-dominated); half of the sample is in the redshift range $0.4 \leq z<0.5$. Table 1 lists the data related to the final sample. Figure 1 displays each source in composite RGB image using $H$, $I$, and $V$ bands (ACS $F 606 W$ filter). As mentioned above, one object in our sample (LTG 21604) does not have ACS coverage, and is thus shown separately in Fig. 2.

\section{Methodology}

In order to prepare the images for Sérsic model fitting, square postage stamp images of $300 \times 300$ pixels for the $H$ band $\left(18^{\prime \prime} \times 18^{\prime \prime}\right)$, and $600 \times 600$ pixels for the $I$ band (same physical size with half pixel scale) were produced from the survey mosaic, updating the headers with the PYTHON package MONTAGE-wrapper ${ }^{2}$. The size of the stamps was chosen to be big enough to account for sufficient sky pixels and to give a good fit of the main galaxy, while being small enough to be fit in reasonable amounts of computing time. Corresponding noise maps of the same size were also cut.

The next step was to run SEXTRACTOR (Bertin \& Arnouts 1996) in order to identify neighboring objects and to create catalogs with values of apparent magnitude, size, axis ratio, and position angle. These were used later as initial guesses in fitting the galaxies' surface brightness profiles with GALFIT (Peng et al. 2002, 2010). By using SEXTRACTOR we minimize the computing time and ease convergence of GALFIT models to the global $\chi^{2}$ minimum. It is worth noting the need of an aperture large enough to capture most of the galaxy light to provide GALFIT with a representative initial guess for the galaxy magnitude. Since our small

\footnotetext{
2 http://montage.ipac.caltech.edu/
}

sample involves closer and better resolved objects, we made several runs with different circular apertures in order to test the influence of the apertures in the estimation of the parameters. We find that for our entire sample we retrieve a more physical set of output parameters when using an aperture of 4 arcsec for the main galaxy, and a smaller one (1 arcsec) for neighboring objects.

Before running GALFIT, masks were created for neighboring sources using the segmentation maps produced with SEXTRACTOR. Nearby bright objects were chosen to be fit simultaneously with the main galaxy in order to remove any light contamination. GALFIT is a state-of-the-art software package that convolves Sérsic 2D models with the point-spread function (PSF), and uses the Levenberg-Marquardt algorithm for error-weighted nonlinear fitting, whereby the $\chi^{2}$ between the PSF-convolved best-fitting model and the observed 2D surface brightness distribution of a galaxy in a given passband are minimized. As the galaxies in our sample are quite extended (up to 16 arcsec in diameter at $H$-band surface brightness of $26 \mathrm{mag} \mathrm{arcsec}^{-2}$ ), we need to use large PSFs (see Sandin 2014, 2015; Buitrago et al. 2017). The PSF of each band was created using the TinyTim HST PSF Modeling tool (Krist et al. 2011), with the maximum sizes available. These PSFs were then rebinned to the current pixel size of our data $\left(0.06^{\prime \prime}\right.$ and $0.03^{\prime \prime}$ for $H$ and $I$ bands, respectively), resulting in final sizes of $\sim 20 \times 20 \operatorname{arcsec}^{2}$ and $\sim 30 \times 30 \operatorname{arcsec}^{2}$. PSF blurring effects are more relevant for galaxy cores (i.e., in the regions of highest intensity) and also for redder bands where the PSF FWHM is broader (Buitrago et al. 2017). In this publication, the authors found that correcting the profiles for the PSF is essential for the study of both the bright cores and fainter components of galaxies at intermediate distances.

We performed single Sérsic fits in order to compare our results with published works in the literature. A 1D Sérsic function (Sérsic 1963) has the form

$I(r)=I_{\mathrm{e}} \exp \left\{-b_{n}\left[\left(\frac{r}{r_{\mathrm{e}}}\right)^{1 / n}-1\right]\right\}$, 


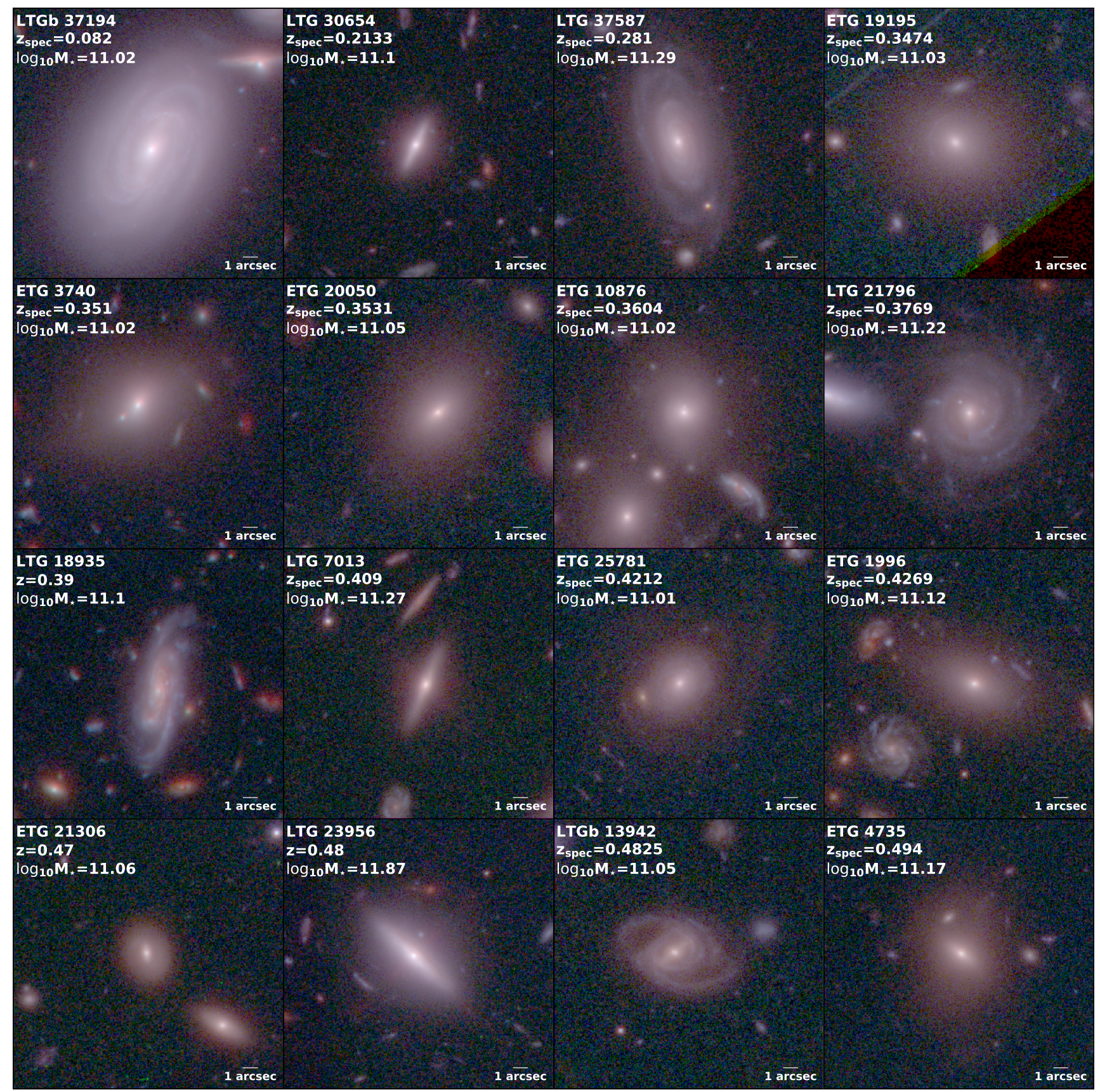

Fig. 1. False-color RGB images of the galaxies within our sample, constructed using the filters $F 160 W, F 814 W$, and $F 606 W$ in the red, green, and blue image channel, respectively. Galaxy 21604 (Fig. 2) is not shown here due to lack of data in the $F 814 W$ and $F 606 W$ filters. Each galaxy stamp contains information about our visual classification preceding the object ID, redshift, and stellar mass. This image compilation illustrates the morphological heterogeneity of our galaxy sample, comprising both ETGs and LTGs, with some of the latter containing a bar.

with $b_{n}$ being a parameter coupled to the Sérsic index $n$ that satisfies the expression $\Gamma(2 n)=2 \gamma\left(2 n, b_{n}\right)$, and where $\Gamma$ and $\gamma$ are the gamma function and the incomplete gamma function, respectively. A Sérsic index of $n=1$ gives an exponential profile, which is commonly assumed to describe perfect exponential galactic disks, whereas $n=4$ yields the de Vaucouleurs law. For a $2 \mathrm{D}$ case (i.e., an astronomical image) the axis ratio and position angle should also be taken into account.

Moreover, $\mathrm{B}+\mathrm{D}$ decompositions were also performed by fitting two Sersic functions, fixing the Sersic index of the disk component to 1 (an exponential). Our aim was not only to describe each galaxy's surface brightness profile as accurately as possible, but also to disentangle the bulge and disk in the case of a LTG, or to check if an ETG is purely elliptical or contains other components (e.g., a disk or bar). Since GALFIT is a least-squares fitting algorithm, the use of bad priors can affect the output parameter values settling the solution into a local $\chi^{2}$ minimum. In the case of single Sérsic fits, the initial parameters do not have a major effect on the fit unless they are considerably different from the actual values. On the other hand, based on extensive tests and experience, we know that for $\mathrm{B}+\mathrm{D}$ decompositions the initial parameters given to GALFIT (especially the magnitudes) are much more important in order to retrieve the models that provide the best residuals. For this reason we adopted different initial parameters for our GALFIT models, according to our visual morphological classification: for ETGs the disk component is set to 


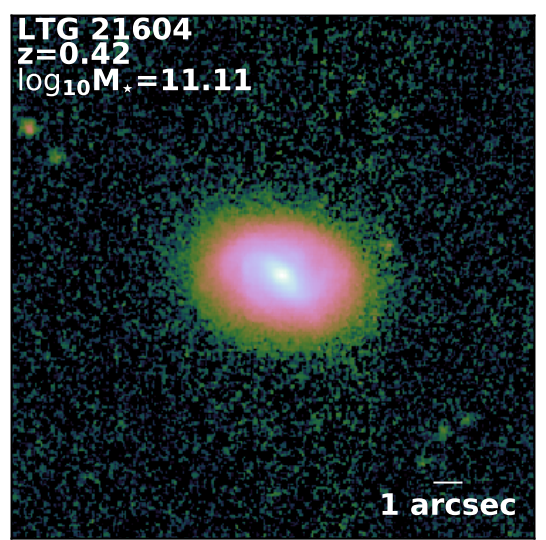

Fig. 2. Galaxy stamp of the late-type object \#21604, as observed in the F160W filter.

be $20 \%$ smaller and 0.5 mag fainter than the spheroid, while in LTGs it is the opposite. For the two galaxies with visual evidence of a bar, an extra component was added to the fit, setting the initial guesses of effective radius and magnitude of the bar with values between the those of the bulge and the disk, the starting Sérsic index value to 0.5 for a flat inner and a steep outer profile, and adding an extra diskiness-boxiness parameter $(C 0$; fixed to 0.5 to ensure a boxy shape). As demonstrated in Gadotti (2008; see also Méndez-Abreu et al. 2008; Breda \& Papaderos 2018), disregarding a bar could contribute to considerable uncertainties in the derived bulge parameters and an overestimation of the bulge-to-total ratio $(B / T)$.

\section{Results and discussion}

In this section we present the main results from our photometric study, both for single Sérsic and multi-component Sérsic fits, for the HST $H$ and $I$ bands. We note that galaxies 13942 and 37194 feature a bar component that was accounted for (both in the single- and the double-component case) through an extra Sérsic component in the fitting procedure. We compare our results with others from the bibliography to check the limitations and advantages of our adopted approach for image decomposition. However, we note that these results from the literature do not take into account the bar component in the fit, whereas our results do.

\subsection{Reliability of the inferred structural parameters}

van der Wel et al. (2012) provides structural parameters from single Sérsic fits of 109533 galaxies within CANDELS in the HST ultra-deep WFC3 $H$ band, which offers a useful database for comparison with our structural analysis. Since our sample is small, we are able to carry out a much more detailed analysis of these low-redshift galaxies (which are thus comparatively large in terms of angular extent). In Fig. 3 we compare the effective radius from our single Sérsic fits with that obtained by van der Wel et al. (2012). To quantitatively assess our comparisons we adopt the widely used normalized median absolute deviation $\left(\sigma_{\text {NMAD }}\right)$, which is equivalent to the standard deviation for a Gaussian distribution with the advantage of being less sensitive to outliers (e.g., Ilbert et al. 2006). The mathematical expression for $\sigma_{\mathrm{NMAD}}$ is the following:

$\sigma_{\mathrm{NMAD}}=1.48 \times \operatorname{median}|X-\operatorname{median}(X)|$.

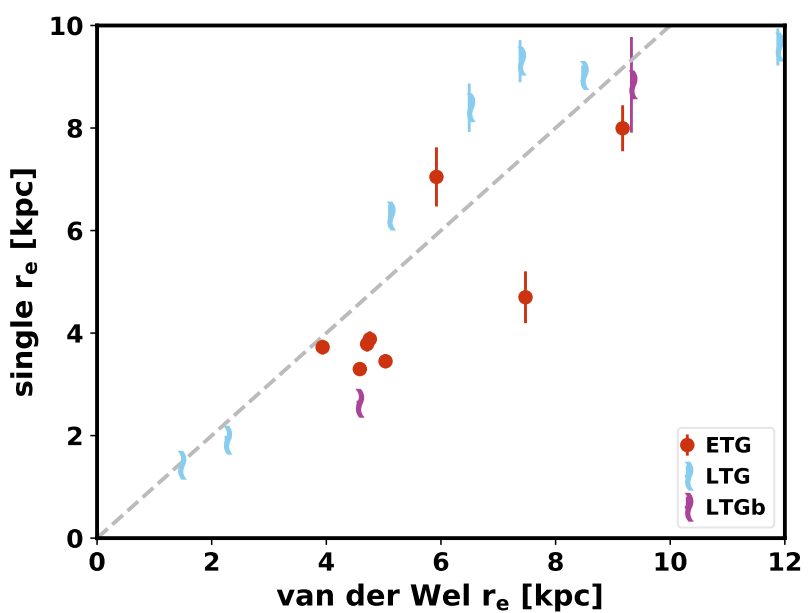

Fig. 3. Comparison between our $H$-band single fit and the van der Wel et al. (2012) effective radius. Our sample is color-coded according to the galaxy visual morphology: red for ETGs, blue for LTGs, and violet for late-type barred galaxies. Error bars come from the simulations described in Appendix A. The dashed line represents the one-to-one relation. Generally our results are in agreement $\left(\sigma_{\mathrm{NMAD}}=0.13\right)$ with van der Wel et al. (2012).

Here $X=\left|\frac{x_{i}-\hat{x}_{i}}{x_{i}}\right|, \hat{x}_{i}$ is our value, and $x_{i}$ is the value with which we are comparing our results. We find good agreement in general, obtaining $\sigma_{\mathrm{NMAD}}=0.13$. The error bars shown for our determinations come from the simulations described in Appendix A.

Figure 4 presents the comparison between our values of the Sérsic index for a single fit and the values obtained by van der Wel et al. (2012), for which we retrieve $\sigma_{\mathrm{NMAD}}=0.10$. In the light of the simulations we present in the appendix and of previous studies in the literature, the discrepancy on some of the recovered Sérsic index values is unsurprising, especially in the case of ETGs. For example, Häussler et al. (2007) tested the performance of two fitting codes (GALFIT and GIM2D) for fitting single Sérsic models for several thousands objects, finding less accurate recovered parameters for ETGs. This is attributed to the fact that these are very concentrated objects and, as such, it is very hard to analyze the inner parts of their surface brightness profiles. For LTGs these discrepancies can be explained by the fact that the SBPs of these galaxies cannot be properly described with a simple Sérsic function, given their structural complexity (e.g., additional bulge and bar, spiral arms, and down-bending disk in some cases).

Another possible explanation for the differences between our results with those from van der Wel et al. (2012), is the PSF we adopted. While we use the same pure TinyTim PSF model for all fields, van der Wel et al. (2012) constructed smaller hybrid PSF models for the different fields, combining stacked stars and TinyTim models. These models were made available in the public release, thus providing us with the possibility to properly compare both results. When using their hybrid PSF we retrieve a much better agreement with van der Wel et al. (2012) structural parameters, confirming that our procedure works as expected, at least for a single Sérsic fit. However, given the brightness and extent of the galaxies in our sample, we use the larger TinyTim PSFs as it will allow us to recover more precise light profiles. Clearly, the sensitivity of the best-fitting solution from GALFIT on the adopted PSF model is a factor that needs to be taken into account when comparing results from different studies.

For the ACS I band it is possible to use the Griffith et al. (2012) catalog, which also includes the fits to the single 


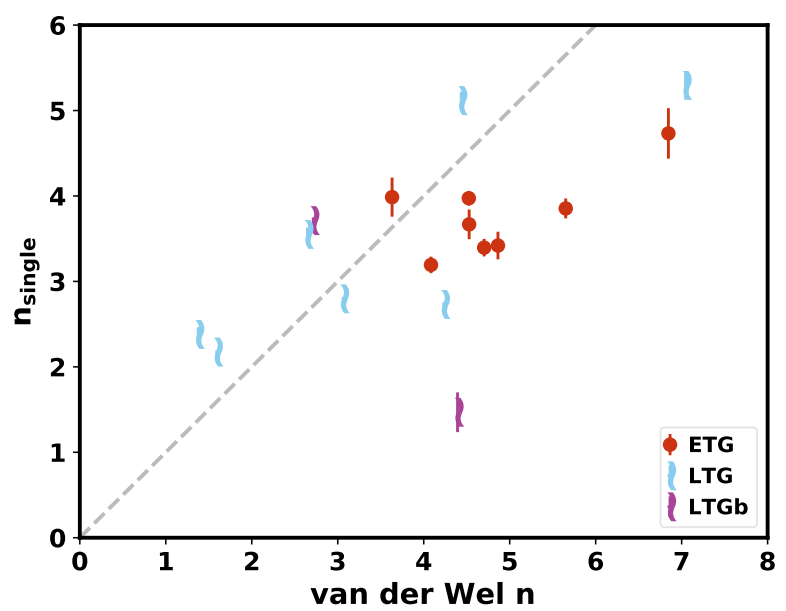

Fig. 4. Comparison between our $H$-band single Sérsic index and that obtained by van der Wel et al. (2012). Our sample is color-coded as in Fig. 3. Error bars come from the simulations described in Appendix A. The divergence in the case of ETGs can be related with their concentrated nature. In the case of LTGs, the discrepancy may be due to the fact that the luminosity profiles of these galaxies cannot be properly described with a simple Sérsic function.

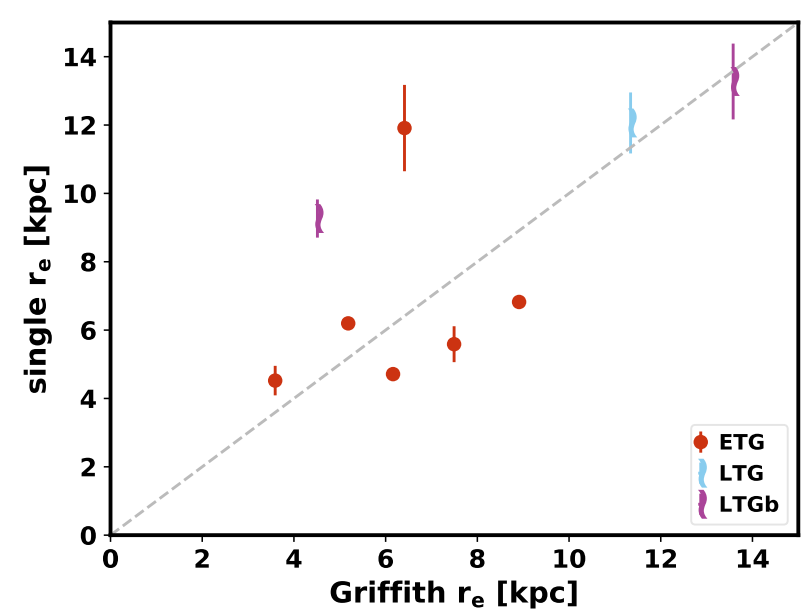

Fig. 5. Comparison between our $I$-band effective radius from single Sérsic fits and the Griffith et al. (2012) effective radius, in kpc. The two objects deviating from the one-to-one line relation are very challenging galaxies to fit in the $I$ band: LTGb 37194 exhibits prominent spiral arms and a nuclear bar, whereas ETG 25781 also appears to have some spiral features evident in the residuals.

Sérsic profiles using GALFIT. Figures 5 and 6 show that our results slightly differ from those in the Griffith et al. (2012) work; we obtain $\sigma_{\mathrm{NMAD}}=0.06$ and $\sigma_{\mathrm{NMAD}}=0.15$ for effective radii and Sérsic indices, respectively. One possible explanation for the disagreement is that their initial conditions for sizes were derived from the formula $r_{\mathrm{e}}=0.162 R_{\text {flux }}^{1.87}$, where $R_{\text {flux }}$ denotes the SEXTRACTOR FLUX_RADIUS, and also the initial condition for their Sérsic index was 2.5. In both cases, their choices are at variance with ours. Furthermore, their study was made automatically for half a million galaxies, making their procedure more susceptible to large uncertainties (e.g., due to an imperfect rejection of overlapping stars or galaxies) that could be eliminated in the case of our far smaller sample. These factors, together with only having nine galaxies in common, may explain the somewhat larger disagreement between our results and Griffith et al. (2012), as opposed to a better agreement with van der Wel et al. (2012).

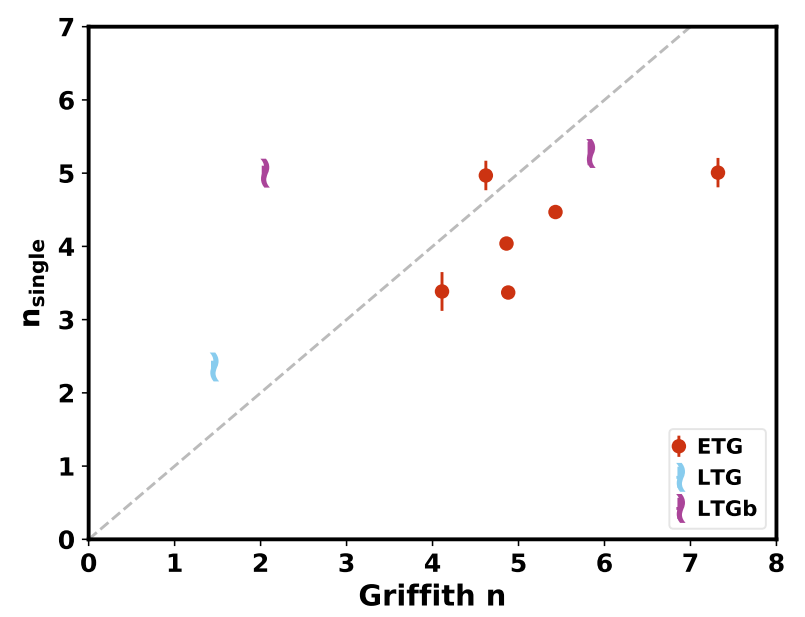

Fig. 6. Comparison between our $I$-band Sérsic index from single Sérsic fits and the Griffith et al. (2012) Sérsic index.

Our GALFIT derived structural parameters for the two photometric bands ( $F 160 W$ and $F 814 W, H$ and $I$ band, respectively) are presented in Tables B.1 and B.2.

\subsection{Sérsic index values}

Figure 7 shows the Sérsic index histograms for the $H$ and $I$ band, in the case of single-component (top panels) and multi-component (bottom panels) fits. For this latter case, we show the Sérsic index values of the spheroidal component ( $\left.n_{\text {spheroid }}\right)$ since the disk component Sérsic index is fixed to 1 (i.e., that corresponding to a pure exponential profile). Following Shen et al. (2003; see also Barden et al. 2005; Trujillo et al. 2006; Buitrago et al. 2013) we use $n=2.5$ as a division line between bulge-dominated and disk-dominated systems, shown in the figure by the dashed gray line. A classification solely based on the Sérsic index would result in only three galaxies classified as disk-dominated $(n<2.5)$ in the $H$ band, and only one in the $I$ band. However, by visual inspection in the $H$ band we classified morphologically nine galaxies as LTGs (diskdominated; shown in the histogram in blue), as opposed to ETGs (bulge-dominated; in red). The bottom panels of Fig. 7 present the Sérsic index for the spheroidal component in the B+D decomposition, the majority of the sample having low $n_{\text {spheroid }}$ values in both bands, which indicates that its light shows a moderate degree of central concentration. These results indicate that visual morphologies and Sérsic morphologies are poorly correlated for our galaxy sample.

\subsection{Bulge-to-total light ratio}

It is well established that the bulge-to-total $(B / T)$ light ratio of a galaxy correlates with its Hubble-type, increasing from LTGs towards ETGs (e.g., Hubble 1926, 1936; Simien \& de Vaucouleurs 1986; Bluck et al. 2014). In Fig. 8 we can see how this quantity behaves with redshift in the two bands being studied. The optical rest frame (bottom panel) and the near-infrared (NIR) rest frame (top panel) both seem to display similar values for each population of galaxies. In the case of LTGs the spheroidal component is not dominant for the majority of these objects $(B / T \leq 0.5)$, except for LTG 7013 which is edge-on and might be a S0 galaxy. In contrast, all ETGs have a dominant spheroidal component $(B / T>0.5)$ for both bands, although the optical rest frame $B / T$ value seems to be slightly higher in all cases, in particular for ETG 21306. 

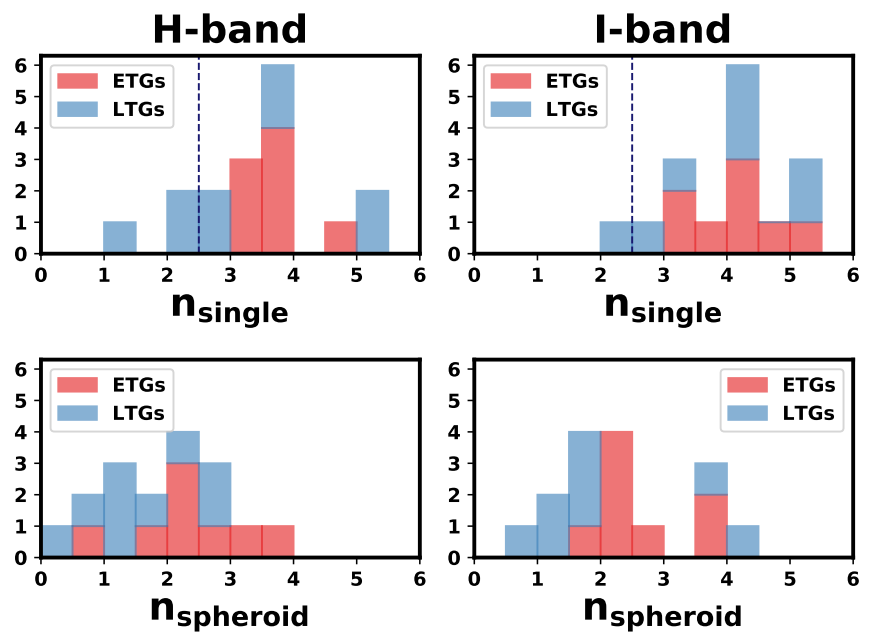

Fig. 7. Sérsic index histograms for single Sérsic fit values (top panels) and for the spheroid component (bottom panels) in the $\mathrm{B}+\mathrm{D}$ decomposition, both for the $H$ (left panels) and the $I$ band (right panels). The dashed gray line represents the division line between disk-dominated $(n<2.5)$ and spheroid-dominated $(n>2.5)$ objects. Our sample contains nine visually classified LTGs, yet single Sérsic fits in the $H$ band yield only three galaxies being classifiable as disk-like on the basis of $n$. Bottom panels: histograms showing that the majority of our sample contains a spheroidal component with a low Sérsic index $\left(n_{\text {spheroid }}<3\right)$ in both bands, pointing to bulges of moderate light concentration.

Three galaxies in our sample have in both bands a $B / T<$ 0.2 , which is frequently linked with pseudo-bulges (Kormendy \& Kennicutt 2004; Kormendy \& Ho 2013). This is thought to reflect assembly in the course of secular galaxy evolution through in situ star formation fed by gas inflow from their disk, inward migration of star-forming clumps, or minor mergers (see, e.g., Scannapieco et al. 2010, see also Breda \& Papaderos 2018 for a recent spectral modeling study of the assembly history of LTG bulges across $\sim 3$ dex in stellar mass). Perhaps it is not surprising that the two barred galaxies in our sample are among these low $B / T$ galaxies. Brook et al. (2012), based on cosmological hydrodynamical simulations, were able to create a disk-like bulge through a bar. However, Kormendy \& Kennicutt (2004) caution that a very low $B / T$ alone does not ensure that the galaxy contains a disk-like bulge; several additional characteristics need to be present, namely a nuclear bar, a boxy shape, and a Sérsic index between 1 and 2 . There also need to be more rotationdominated bulges than classical bulges, and a low-sigma outlier in the Faber \& Jackson (1976) correlation. In addition, the galaxy should be dominated by young stars, gas, and dust but with no evidence of ongoing mergers. For our small albeit meaningful sample, we see no trend between the $B / T$ ratio and redshift.

\subsection{Size estimation}

Since for our high $\mathrm{S} / \mathrm{N}$ galaxy sample a two-component fit provides a better match to the $2 \mathrm{D}$ surface brightness profiles than a single Sérsic model, this double Sérsic model is expected to yield more accurate effective radii. Following the definition of the effective radius $r_{\mathrm{e}}$, we computed curves of growth for the best-fitting two-component GALFIT models by integrating the flux within concentric elliptical apertures until half of the galaxy total flux was reached. The axis ratio and position angle of these ellipses is fixed to the values of each galaxy's single Sérsic models. In order to check the accuracy of our algorithm,
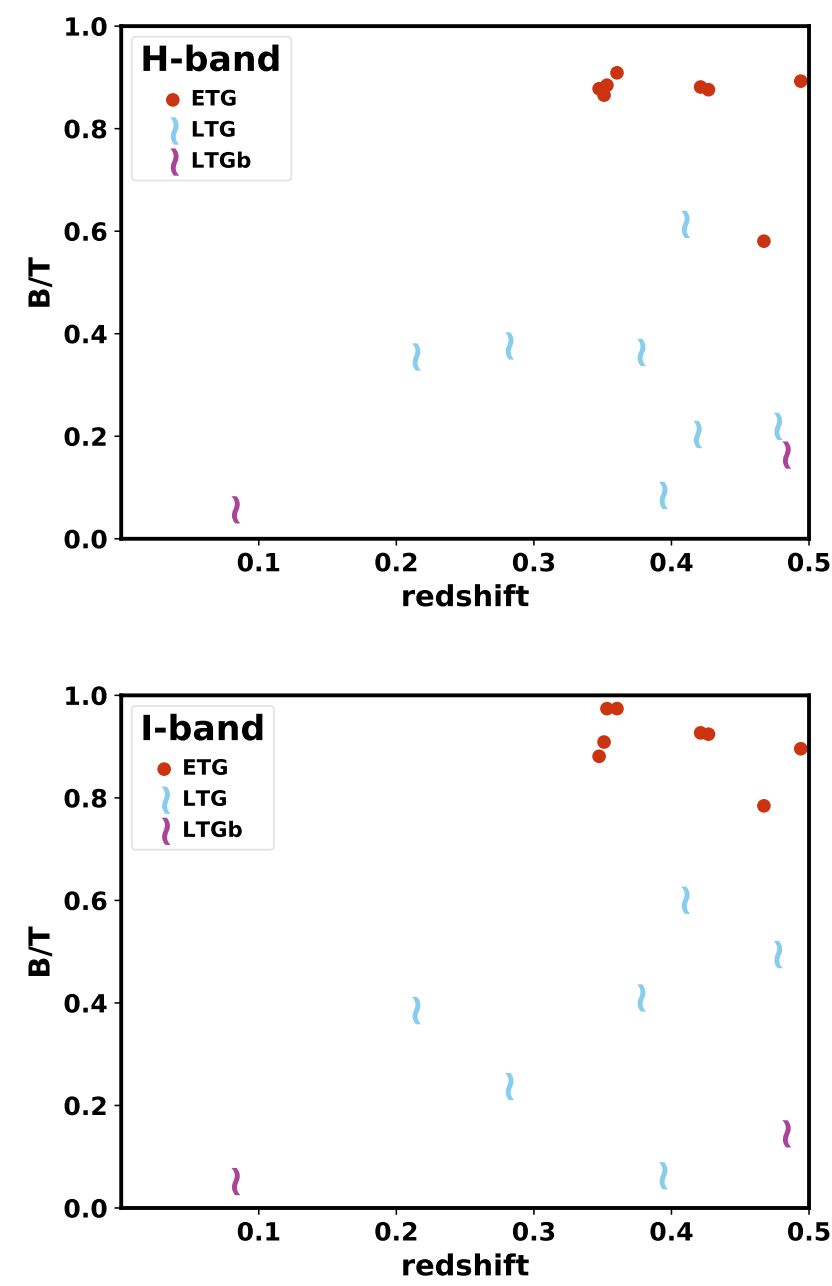

Fig. 8. Bulge-to-total $(B / T)$ light ratio relation with redshift for $H$ band (top panel) and $I$ band (bottom panel). The $B / T$ values do not change significantly between the $H$ band (NIR rest frame) and the $I$ band (optical rest frame) for LTGs, being mostly disk-dominated $(B / T \leq 0.5)$, with exception of LTG 7013 which is an edge-on galaxy and visually seems to be an S0 galaxy. In contrast, for ETGs the bulge component seems to dominate $(B / T>0.5)$ in a similar way in both bands, being the optical rest frame slightly higher in all cases but particularly for ETG 21306.

the same scheme was applied to single Sérsic models, yielding a satisfactory agreement between the $r_{\mathrm{e}}$ obtained with the curves of growth and the $r_{\mathrm{e}}$ obtained directly from the GALFIT output.

In Fig. 9 we show the relation between the single-component and the computed multi-component effective radius in the two bands. Most of the deviating objects with the single Sérsic models implying a higher $r_{\mathrm{e}}$ than multi-component Sérsic fits are LTGs. This trend can be explained by the fact that these objects require using a combination of at least two models of light (disk + spheroid) for an adequate study of their structures. In the case of ETGs, this effect could also be related to imposing an exponential disk in a multi-component Sérsic fit of a galaxy which does not require such a component. To test this we performed a new multi-component analysis of each earlytype galaxy, leaving the two Sérsic index values free. The results from this test did not appreciably change the values of the effective radius, leaving us with the conclusion that the assumption of a disk $(n=1)$ instead of an additional higher- $n$ Sérsic component does not appreciably alter $r_{\mathrm{e}}$ determinations for ETGs with 

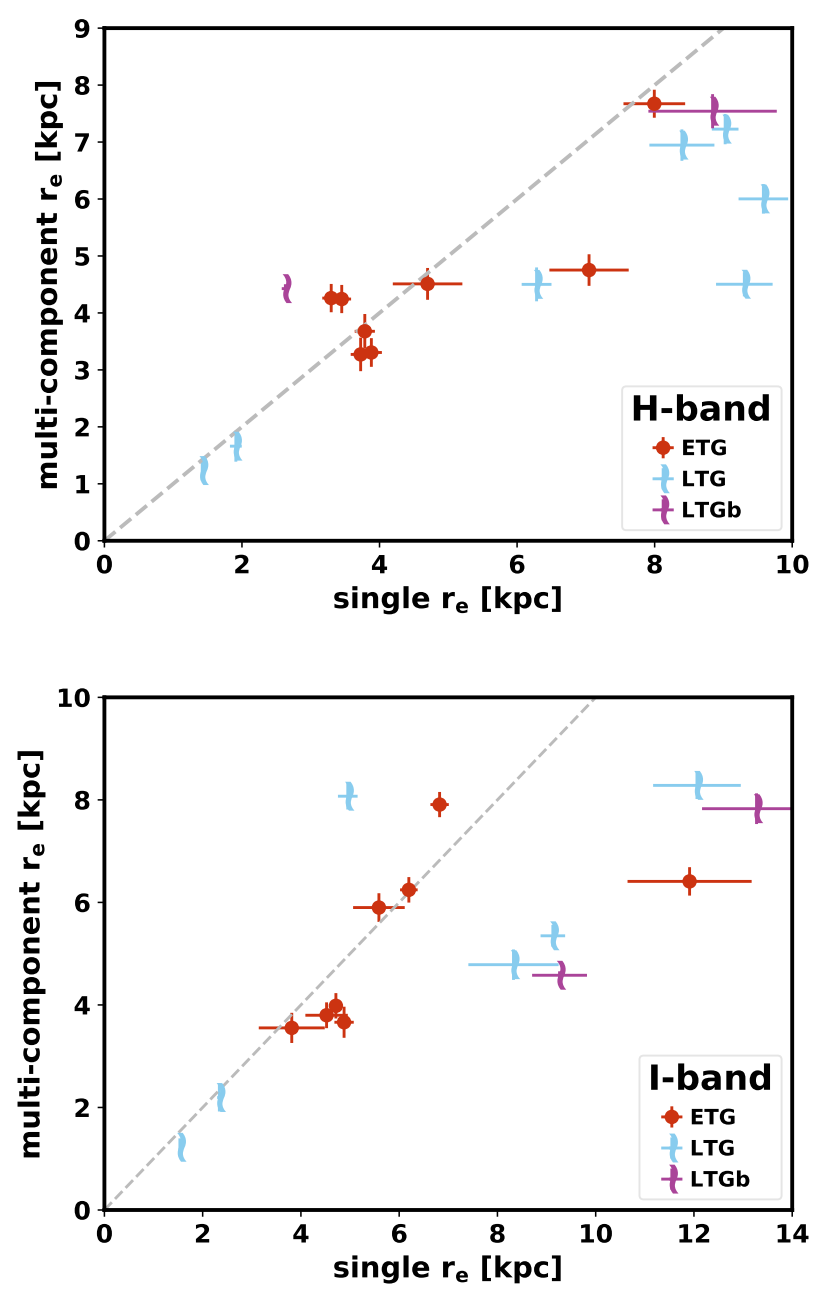

Fig. 9. Relation between single- and multi-component effective radius in kpc, for the $H$ band (top panel) and for the $I$ band (bottom panel).

GALFIT. We think a two-component analysis is always a better representation of a galaxy surface brightness profile, and so this plot indicates that high single Sérsic effective radii are not accurate proxies of galaxy sizes, and caution needs to be taken when interpreting effective radii greater than $10 \mathrm{kpc}$ from single Sérsic fits.

Figure 10 shows our computed multi-component effective radii for both bands. We retrieve a good correlation (values close to the one-to-one line relation, together with $\sigma_{\mathrm{NMAD}}=0.12$ ) between the computed sizes in the different bands, yet systematically obtaining slightly higher values for the $I$ band.

\subsection{Mass-size relation}

The relation between galaxy size (usually quantified as $r_{\mathrm{e}}$ ) and mass evolves differently for spheroid-like and disk-like massive galaxies, with spheroids having a stronger evolution in size than disk-like objects (e.g., Trujillo et al. 2007; Buitrago et al. 2008; van der Wel et al. 2014).

Shen et al. (2003) investigated the size distribution of $\sim 140000$ galaxies from the SDSS and its dependence on luminosity, stellar mass, and morphology, separating spheroiddominated and disk-dominated systems accordingly with the indicators $n=2.5$ and $c=2.86$, which refer to the Sérsic index and concentration index (see Shimasaku et al. 2001), respectively. In another work, van der Wel et al. (2014) took advantage

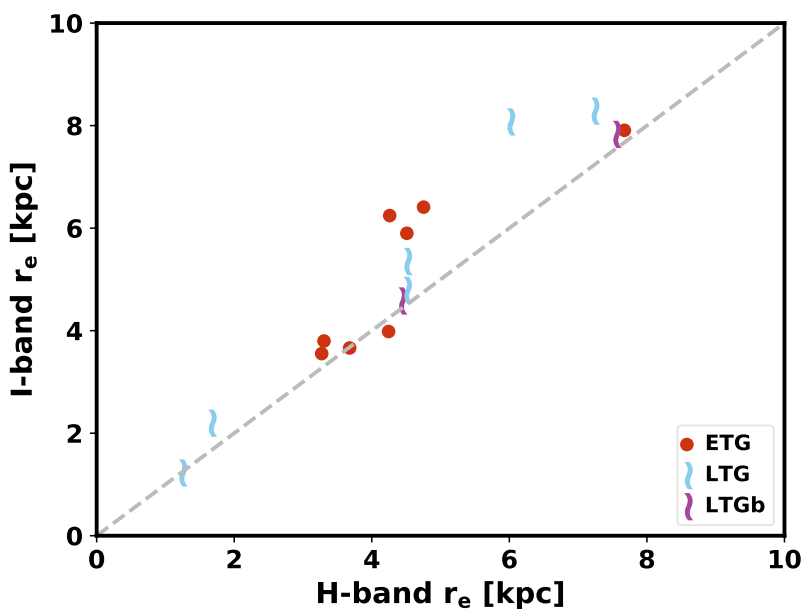

Fig. 10. Comparison between the size estimates (computed multicomponent effective radii) in units of kpc, using the WFC $3 H$-band vs. ACS $I$-band filters, for 16 galaxies in our sample. There is a good correlation between the computed sizes in both bands, although with a tendency for slightly higher values for the $I$ band.

of the slitless spectroscopy from the 3D-HST survey combined with CANDELS photometry to study the evolution of the galaxy size-mass distribution since $z=3$. The authors separate the two classes of galaxies on the basis of star formation activity, using rest frame $U-V$ and $V-J$ color distributions of galaxies with stellar mass above $10^{10} M_{\odot}$. The combination of these two works is the reference we utilize to compare our results with.

We construct the mass-size relation for our $H$ - and $I$-band data in Figs. 11 and 12, and compare them with the results from Shen et al. (2003), and van der Wel et al. (2014) for their lowest redshift bin centered at $z=0.25$. We highlight (purple axis) the plot we consider most relevant, which is the one that has the most representative effective radius (multi-component $r_{\mathrm{e}}$, and not circularized as in the works we use for comparison). The circularized effective radius $\left(r_{\mathrm{e}, \mathrm{circ}}=r_{\mathrm{e}} \sqrt{b / a}\right)$ is commonly used in the literature to correct for the line-of-sight projection of triaxial ETGs, and thus we display our results both with and without circularization (right and left panels, respectively). It should be noted, even though the median redshift of our sample is 0.39 , that our results are consistent with those found by Shen et al. (2003), having almost the entire sample falling inside their scatter. This similarity between the two mass-size relations indicates that these galaxies do not experience a large evolution in sizes. Furthermore, in both bands we find two LTGs that are outliers (with sizes $\sim 2 \sigma$ below the Shen et al. 2003 relation), implying that ETGs already seem to be in place early on.

\subsection{Surface brightness profiles and color profiles}

To check how the observed surface brightness profiles of our sample galaxies compare with those implied by the best-fitting models with GALFIT we computed from the latter 1D SBPs for both $H$ and $I$ band. The profiles for the entire sample can be found in Appendix C.

In Fig. 13 we present SBPs for three representative galaxies from our sample that were visually classified as ETGs (top panels), barred LTGs (middle panels), and LTGs (bottom panels). The profiles in the $H$ band and $I$ band are displayed in the left and right panel, respectively, and for both single- and multi-component Sérsic models. The inset in each profile contains the corresponding galaxy stamp in units of surface brightness (mag $\operatorname{arcsec}^{-2}$; see 

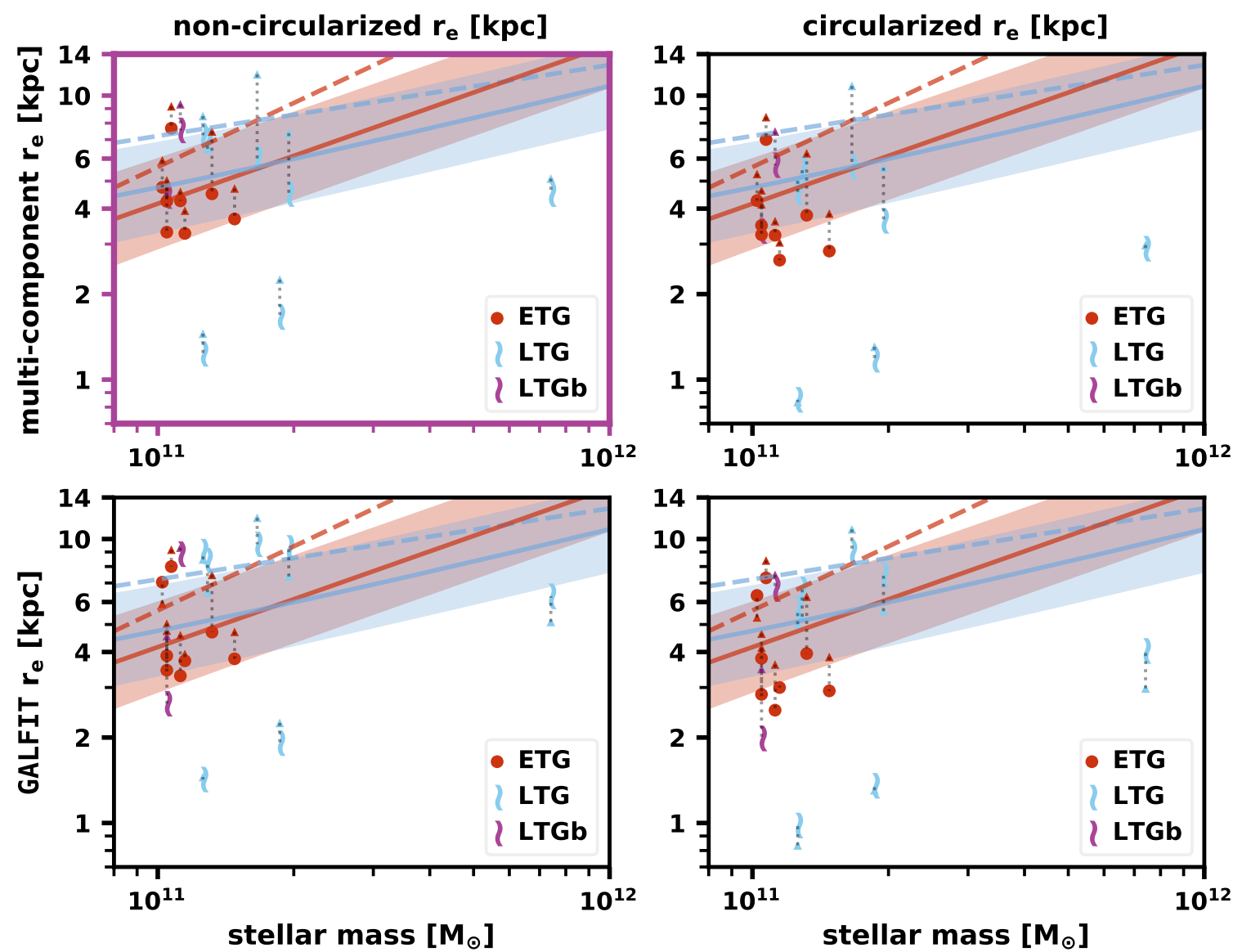

Fig. 11. Size-stellar mass distribution of our $H$-band data, linked to van der Wel et al. (2012) results represented by triangles. Left panels: noncircularized effective radii, while the right panels show the circularized ones $\left(r_{\mathrm{e}, \mathrm{circ}}\right)$. Top panels: correspond to our computed multi-component effective radii, whereas the bottom panels show the GALFIT single Sérsic effective radii. The Shen et al. (2003) local relation for ETGs and LTGs is represented by the solid red and blue lines, respectively, with the corresponding scatter being the shaded red and blue regions. The colored dashed lines correspond to the van der Wel et al. (2014) mass-size relations at $z=0.25$, as obtained from noncircularized $r_{\mathrm{e}}$ determinations. Our results lie within the scatter of Shen et al. (2003) local relation. We choose to highlight in purple the axis of our preferred plot since it shows the values of the most representative effective radius (multi-component $r_{\mathrm{e}}$ ) without circularization (as in the works that we use for comparison).

the corresponding color bar) with shadowed areas matching the masks used to recover the observed light profile.

All galaxies in our sample are more luminous in the $H$ band. For almost half of our sample an overprediction of the light in the galaxy outskirts is obtained when performing a single Sérsic fit (solid purple line). For the rest of the objects the light is underestimated in the outskirts, both for the single Sérsic fit and the multi-component fit (solid green line). As expected, multicomponent Sérsic fits yield a better match to the observed SBPs (black points).

Despite our efforts with $\mathrm{B}+\mathrm{D}$ decompositions, many objects show small-scale patterns in their residuals in both bands after the removal of the principal galaxy components (bulge plus disk). These residuals hold clues on galaxy substructures that cannot be described by axisymmetric Sérsic functions, although their physical interpretation is not always clear. These subtle features are listed below, and are included as a figure in Appendix E:

- LTGb 37194 shows large over-subtractions surrounding the entire galaxy, but also between the spiral arms.

- LTG 30654 has symmetric over-subtractions on both sides from the center of the disk plane, perpendicular to the minor axis of the galaxy that stand out as a nuclear stellar disk.

- LTG 37587 exposes its spiral arms with some oversubtractions around them.
- ETG3740 displays asymmetric over- and undersubtractions in the galactic center, suggesting the presence of a faint halo enclosing the galaxy, and a nuclear disk or bar.

- ETG 19195 shows faint circumnuclear rings.

- ETG 20050 shows asymmetric over-subtractions along the galaxy plane, suggesting the presence of a nuclear bar with lowlevel dust patterns.

- ETG 10876 reveals nuclear spiral arms with asymmetric over-subtractions, and a nuclear point source.

- LTG 21796 exhibits asymmetric over- and undersubtractions, highlighting some spiral patterns and two point sources on each side of the nucleus.

- LTG 18935 has considerable over- and under-subtractions that stand out in its spiral arms and clumps.

- LTG 21604 reveals an interesting asymmetric luminous central substructure with spiral-like features.

- ETG 25781 exposes very faint circular rings.

- ETG 1996 displays a small under-subtraction in the galactic center.

- ETG 21306 presents an asymmetric under-subtraction in the outskirts of the galaxy, suggesting the presence of an outer asymmetric envelope.

- ETG 4735 has symmetric over-subtractions perpendicular to the major axis of the galaxy, on both sides from the center 

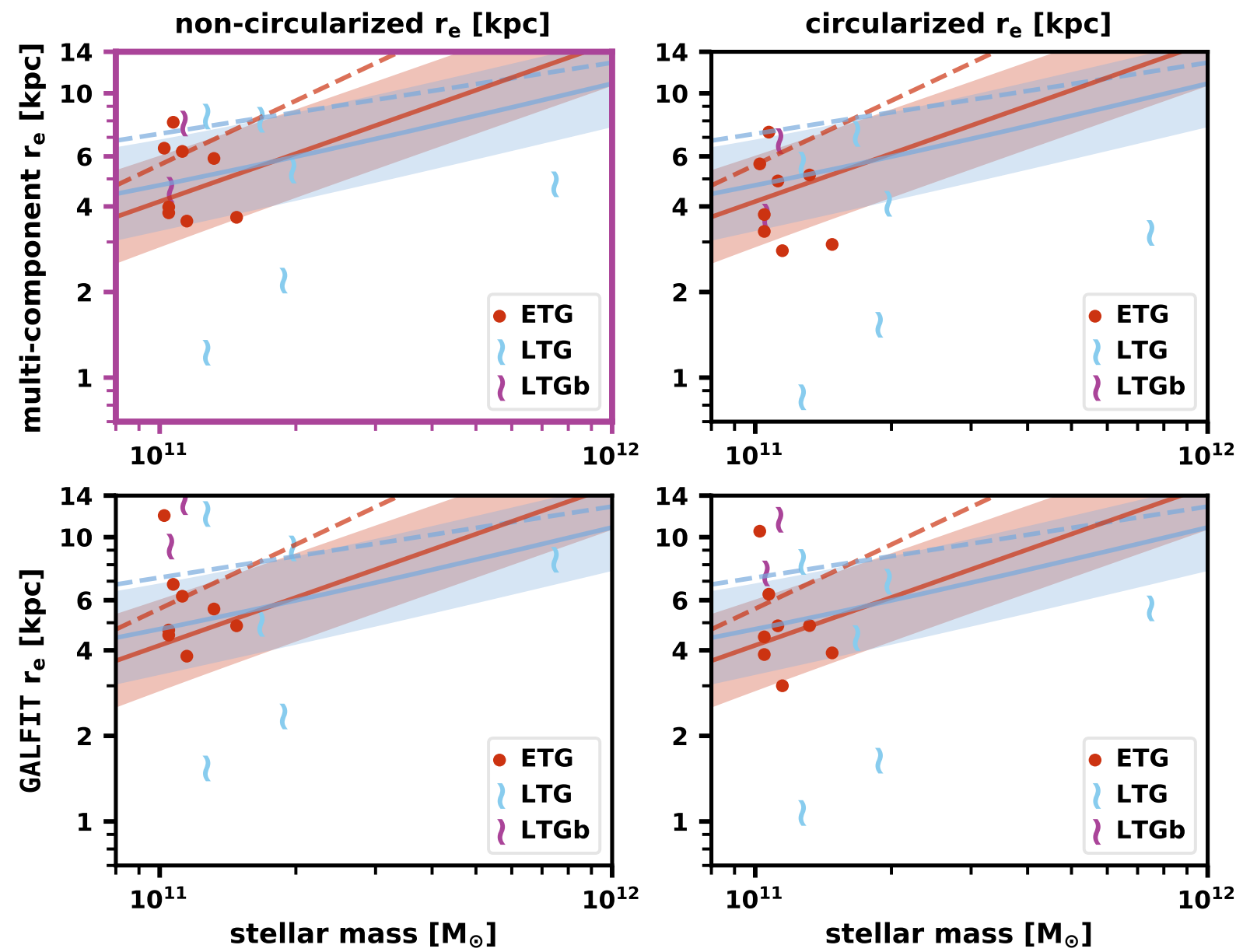

Fig. 12. Size-stellar mass distribution of our $I$-band data (see the caption of Fig. 11 for a detailed description of this image). Similar to the results of the $H$ band, most of our sample lies in the local relation of Shen et al. (2003), with the exception of the same two LTGs, which are around $\sim 2 \sigma$ smaller than expected.

of the disk plane, appearing to be a disk but likely an artifact created by a model mismatch.

- LTG 23956 displays asymmetric over-subtractions in the central part of the galaxy, highlighting a nuclear substructure with dust patterns, and a faint halo surrounding the outer parts of the galaxy.

- LTG7013 exhibits symmetric cross-shaped oversubtractions.

- LTGb 13942 exposes spiral arms in the outer parts of the disk, a circumnuclear ring, and another ring around the bar component.

Visual inspection of the residuals after subtraction of the best-fitting 2D model from an image clearly reveals large contiguous regions where the fit over- or underestimates the galaxy surface brightness; however, this comparison does not allow a quantitative inference on these residuals in units of mag. Having this information would be important, however, since among other things it is also needed for estimating the goodness of the color maps implied by subtraction of the best-fitting 2D model with GALFIT in two different bands. An inference on such deviations in mag can, however, be more easily obtained from comparison of synthetic and observed SBPs in Fig. 14 and in the figures of Appendix D. It can be seen, especially in the periphery of galaxies, that the fit in many cases deviates by $0.5-1 \mathrm{mag}$ from the observed SBP, both for single and double Sérsic fits. This documents a global failure of the adopted fitting scheme with GALFIT to give a proper approximation to the surface brightness (and color) of the low surface brightness outskirts of the galaxies under study. Therefore, the extensive set of simulations on mock galaxy images (Appendix A) demonstrates, on the statistical average, that GALFIT can retrieve Sérsic model parameters with a satisfactory accuracy; however, individual fits can yield systematic and substantial deviations from the true 1D-2D surface brightness distribution of even for a relatively "simple" galaxy like an ETG. This may be partly attributed to the inherent uncertainties in nonlinear fitting, which, as empirically quantified in Appendix A, increase with increasing Sérsic exponent and decreasing luminosity (i.e., $\mathrm{S} / \mathrm{N}$ ).

Furthermore, the specifics of the fitting procedure could also strongly influence Sérsic model solutions. For example, Papaderos et al. (1996) used the Levenberg-Marquardt nonlinear fitting algorithm to decompose SBPs of blue compact dwarf galaxies into a Sérsic and a Gaussian component, accounting for the luminosity contribution of the star-forming component, plus an exponential component approximating the more extended underlying stellar host. They pointed out that the solution significantly depends on whether or not SBP data points are weighted by photometric uncertainties $\sigma \mu_{\mathrm{i}}$ (because central points have lower uncertainties); doing so generally leads to the innermost (highest surface brightness and thus lowest $-\sigma \mu_{\mathrm{i}}$ points) dictating the solution, which typically leads to a "compactification" of the exponential host in the sense of an overestimated central 

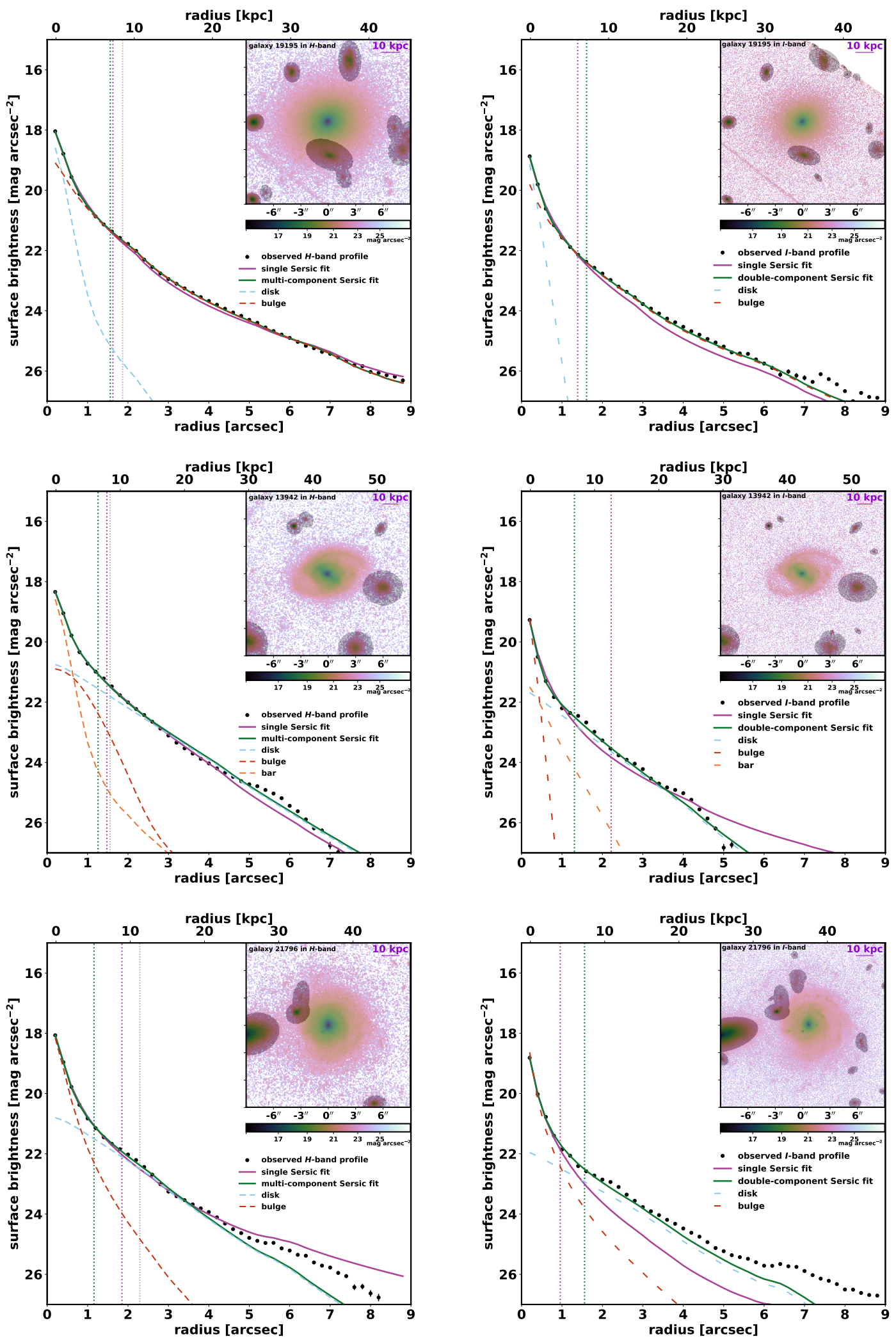

Fig. 13. Surface brightness profiles in the $H$ band (left panels) and in the $I$ band (right panels) for three systems illustrating the different galaxy morphologies in our sample: the early-type galaxy 19195 (top panels), the barred late-type galaxy 13942 (middle panels), and the late-type galaxy 21796 (bottom panels). The results from single- and multi-component Sérsic models are shown in violet and green, respectively. Black points represent the observed surface brightness profile; solid lines show the models convolved with the PSF; dashed lines stand for the decomposition of the multi-component model into bulge (red), disk (blue), and bar (orange); dotted vertical lines represent the effective radius; and the dotted gray vertical line is the van der Wel et al. (2012) (single Sérsic) effective radius. We display the galaxy stamp in units of surface brightness (mag $\operatorname{arcsec}^{-2}$; see the horizontal color bar beneath each map) with shadowed areas matching the masks used to obtain the observed light profile, the galaxy ID in the top left corner, and a scale bar in the top right corresponding to $10 \mathrm{kpc}$. 

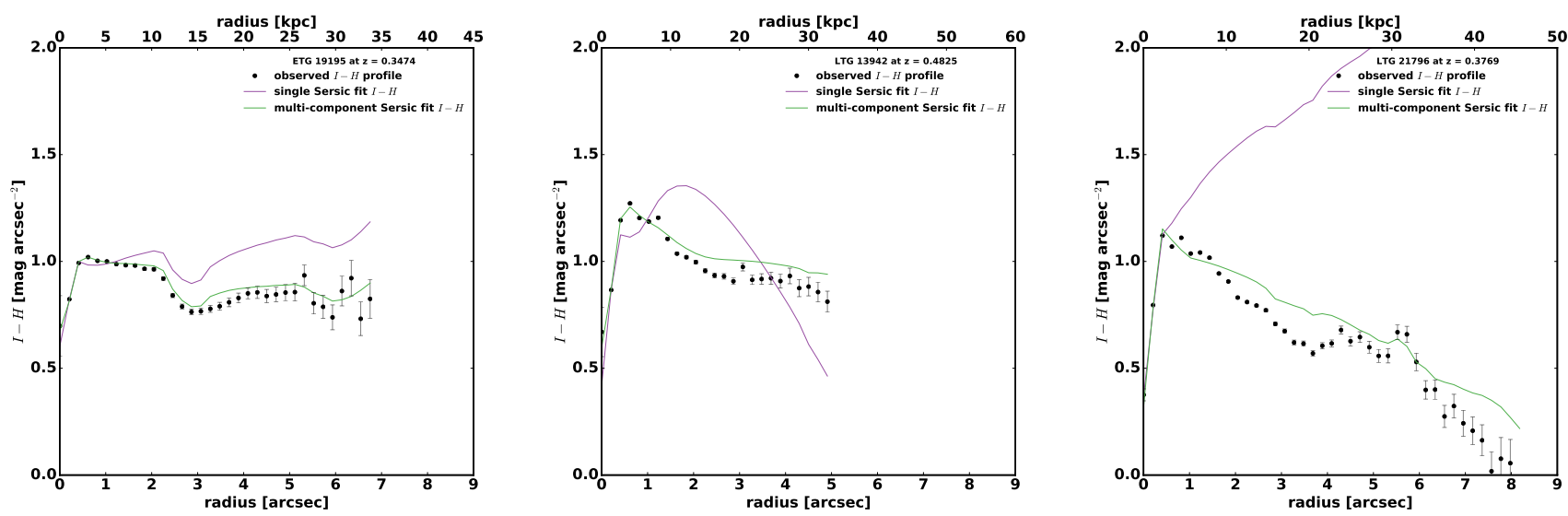

Fig. 14. $I-H$ color profiles for ETG 19195, LTGb 13942, and LTG 21796. Data points with larger error bars (>0.3 mag) are not shown.

surface brightness and underestimated exponential scale length ${ }^{3}$. This may also reflect on the rest of the structural parameters (see also Ribeiro et al. 2016).

The dependence of the fit on the central data points makes a precise correction for PSF convolution effects critically important and eventually partly accounts for the systematic deviations between the best-fitting Sérsic model and the observed SBP in the low surface brightness periphery of galaxies. Furthermore, at this depth and for the case of ETGs, we may be looking at stellar haloes being both structurally and evolutionary distinct from the galaxy main body. Such deviations are apparent from several GALFIT models, and more noticeable when producing the $I-H$ color profiles implied by subtraction of GALFIT models in the two different bands (see Fig. 14 and Appendix D). This is an elementary consistency check for any 1D or 2D parametric fitting approach, namely whether the color profiles implied by fits in individual bands are in reasonable agreement with the observed ones and make sense from the astrophysical point of view. The fulfillment of the latter condition is obviously crucial to ensure that the adopted 1D-2D parametric fitting procedure leads to meaningful observational constraints on the age distribution of stellar populations in galaxies. As is clear from Fig. D.1, this is not the case for most galaxies under study, given that deviations of $>0.3$ mag between observed and modeled color profiles are apparent over extended zones, both for multi- and single-component Sérsic fits ( 7 and 3 of the 13 profiles, respectively). In the case of the ETGs in our sample, the extra light at larger galactocentric distances is most probably due to their haloes. Nevertheless, CANDELS is not optimized to study the low surface brightness Universe, which means that our images could be potentially affected by the way their data reduction was performed (see Buitrago et al. 2017; Borlaff et al. 2019).

\section{Summary and conclusions}

We accomplished a photometric study of the 17 low-redshift $(z<0.5$, with 13 spectroscopic redshifts and a median redshift of 0.39$)$ massive galaxies $\left(M_{\text {stellar }} \geq 10^{11} M_{\odot}\right)$ in the CANDELS fields. Using superb-quality imaging data from a survey intended for high-redshift science provides an unprecedented photometric depth and spatial resolution. This information was complemented with the slitless spectroscopy data in the 3D-HST survey.

\footnotetext{
3 For this reason, these authors considered both weighted and unweighted nonlinear fitting solutions in their analysis.
}

We obtained single Sérsic fits to compare with previous works in the literature, namely van der Wel et al. (2012) for the $H$ band and Griffith et al. (2012) for the $I$ band. In general we find good agreement with their results, suggesting that a detailed modeling of individual galaxies with GALFIT yields a rather satisfactory agreement with results obtained from automated application of the code to large galaxy samples.

We performed $\mathrm{B}+\mathrm{D}$ decompositions on our entire sample in the $H$ and $I$ bands, deriving multi-component effective radii for each galaxy, their surface brightness profiles, and constructed a mass-size relation for comparison with standard published references. Our results indicate that morphology classifications based on single Sérsic fitting are poorly correlated with the visual morphology of our galaxy sample population. In particular, by using the division line of $n=2.5$ to differentiate spheroid-dominated from disk-dominated systems in the $H$ band, we would have only three galaxies classified as disk-like, whereas nine LTGs were identified in our sample on the basis of visual classification. Regarding galaxy sizes, by computing more accurate multi-component effective radii, we show that they systematically deliver small sizes than single Sérsic effective radii when the values for the latter are greater than $10 \mathrm{kpc}$.

From the constructed mass-size relation, we located most of the galaxies in our sample within the scatter of the local Shen et al. (2003) mass-size relation, indicating that these galaxies have a small evolution in sizes since $z<0.5$. Moreover, the two detected outliers in the relation are disk-like galaxies, implying that spheroids are already at place early on.

Although GALFIT generally gives satisfactory fits (in terms of $\chi^{2}$ minimization) to galaxy images in individual bands, we find that the best-fitting Sérsic model can substantially and systematically deviate from observed surface brightness profiles at intermediate to low surface brightness levels. This issue probably arises from the fact that the global solution in profile fitting and decomposition primarily depends on the innermost regions (highest $\mathrm{S} / \mathrm{N}$ yet most affected by the PSF) of a galaxy. Additionally, in the case of the deep observations we are dealing with, these deviations could be partly due to the extended stellar halo of ETGs. We also note that a data reduction optimized for the preservation of low surface brightness features is mandatory for the next generation surveys to also be able to utilize their imaging for the $z<1$ Universe. Regardless of its origin, the failure of parametric $2 \mathrm{D}$ decomposition with GALFIT to closely fit the low surface brightness periphery in more than one half of the galaxies in our sample is a significant concern. One of the implications is that color profiles constructed from subtraction of 2D models in 
two different bands may differ from observed profiles by more than one mag. A consequence is that studies of the inside-out growth of galaxies across redshift that are based on synthetic color profiles from GALFIT could be subject to unpredictable systematic uncertainties.

Acknowledgements. We would like to thank the anonymous referee for valuable comments and suggestions. We thank the European taxpayer, who in the spirit of solidarity and mutual respect between EU countries provides the Fundação para a Ciência e a Tecnologia (FCT) with a substantial fraction of the financial resources that allow it to sustain a research infrastructure in astrophysics in Portugal. Specifically, this work was supported by FCT through national funds and by FEDER through COMPETE by the grants UID/FIS/04434/2013 \& POCI-01-0145FEDER-007672 and PTDC/FIS-AST/3214/2012 \& FCOMP-01-0124-FEDER029170. Additionally, we acknowledge support by FCT/MCTES through national funds (PIDDAC) by grant UID/FIS/04434/2019. We thank all members of the Thematic Line "The assembly history of galaxies resolved in space and time" of Instituto de Astrofísica e Ciências do Espaço (IA) for a critical review of the manuscript and numerous valuable comments and suggestions. FB acknowledges the support by FCT via the postdoctoral fellowship SFRH/BPD/103958/2014, the conversations with the TRACES group of galaxy evolution in the IAC, and also the RAVET programme. PP acknowledges support by FCT through Investigador FCT contract IF/01220/2013/CP1191/CT0002, as well as support by FCT/MCTES through national funds (PIDDAC) by PTDC/FISAST $/ 29245 / 2017$. This project has benefited from support by European Community Programme (FP7/2007-2013) under grant agreement No. PIRSESGA-2013-612701 (SELGIFS). I.B. was supported by the FCT PhD::SPACE Doctoral Network (PD/00040/2012) through the fellowship PD/BD/52707/2014 funded by FCT (Portugal) and POPH/FSE (EC) and by the fellowship CAUP07/2014-BI in the context of the FCT project PTDC/FIS-AST/3214/2012 \& FCOMP-01-0124-FEDER-029170, as well as by the fellowship with reference CIAAUP-19/2019-BIM within the scope of the research unit IA. J.M.G. is supported by the DL 57/2016/CP1364/CT0003 contract and acknowledges the previous support by the fellowships CIAAUP-04/2016-BPD in the context of the FCT project UID/-FIS/04434/2013 \& POCI-010145-FEDER-007672, and SFRH/BPD/66958/2009 funded by FCT and POPH/FSE(EC). This work is based on observations taken by the CANDELS Multi-Cycle Treasury Program with the NASA/ESA HST, which is operated by the Association of Universities for Research in Astronomy, Inc., under NASA contract NAS5-26555. We acknowledge the use of the following software packages: TOPCAT (Taylor 2005), ALADIN (Bonnarel et al. 2000), SEXTRACTOR (Bertin \& Arnouts 1996), TinyTim HST PSF Modeling (Krist et al. 2011). This research has been partly supported by the grant AYA-2016-77237-C3-1-P from the Spanish Ministry of Science, Innovation and Universities. This research made use of MONTAGE. It is funded by the National Science Foundation under Grant Number ACI-1440620, and was previously funded by the National Aeronautics and Space Administration's Earth Science Technology Office, Computation Technologies Project, under Cooperative Agreement Number NCC5-626 between NASA and the California Institute of Technology. It also made use of the following PYTHON packages: NumPy \& SciPy (Jones et al. 2001), Matplotlib (Hunter 2007), Astropy (Astropy Collaboration 2013), a community-developed core Python package for Astronomy; APLpy (Robitaille \& Bressert 2012), an open-source plotting package.

\section{References}

Allen, P. D., Driver, S. P., Graham, A. W., et al. 2006, MNRAS, 371, 2 Astropy Collaboration (Robitaille, T. P., et al.) 2013, A\&A, 558, A33 Barden, M., Rix, H.-W., Somerville, R. S., et al. 2005, ApJ, 635, 959 Bertin, E., \& Arnouts, S. 1996, A\&AS, 117, 393

Bezanson, R., van Dokkum, P. G., Tal, T., et al. 2009, ApJ, 697, 1290 Bluck, A. F. L., Conselice, C. J., Buitrago, F., et al. 2012, ApJ, 747, 34 Bluck, A. F. L., Mendel, J. T., Ellison, S. L., et al. 2014, MNRAS, 441, 599 Bonnarel, F., Fernique, P., Bienaymé, O., et al. 2000, A\&AS, 143, 33 Borlaff, A., Trujillo, I., Román, J., et al. 2019, A\&A, 621, A133 Breda, I., \& Papaderos, P. 2018, A\&A, 614, A48 Brook, C. B., Stinson, G., Gibson, B. K., et al. 2012, MNRAS, 419, 771 Buitrago, F., Trujillo, I., Conselice, C. J., et al. 2008, ApJ, 687, L61 Buitrago, F., Trujillo, I., Conselice, C. J., \& Häußler, B. 2013, MNRAS, 428, 1460

Buitrago, F., Trujillo, I., Curtis-Lake, E., et al. 2017, MNRAS, 466, 4888 Damjanov, I., McCarthy, P. J., Abraham, R. G., et al. 2009, ApJ, 695, 101 de la Rosa, I. G., La Barbera, F., Ferreras, I., et al. 2016, MNRAS, 457, 1916 de Vaucouleurs, G. 1948, Ann. Astrophys., 11, 247

Dimauro, P., Huertas-Company, M., Daddi, E., et al. 2018, MNRAS, 478, 5410 Djorgovski, S., \& Davis, M. 1987, ApJ, 313, 59

Faber, S. M., \& Jackson, R. E. 1976, ApJ, 204, 668

Ferreras, I., Trujillo, I., Mármol-Queraltó, E., et al. 2014, MNRAS, 444, 906 Ferreras, I., Hopkins, A. M., Gunawardhana, M. L. P., et al. 2017, MNRAS, 468, 607

Freeman, K. C. 1970, ApJ, 160, 811

Gadotti, D. A. 2008, MNRAS, 384, 420

Gomes, J. M., Papaderos, P., Vílchez, J. M., et al. 2016, A\&A, 585, A92

Griffith, R. L., Cooper, M. C., Newman, J. A., et al. 2012, VizieR Online Data Catalog: II/200

Grogin, N. A., Kocevski, D. D., Faber, S. M., et al. 2011, ApJS, 197, 35

Häussler, B., McIntosh, D. H., Barden, M., et al. 2007, ApJS, 172, 615

Hopkins, P. F., Bundy, K., Murray, N., et al. 2009, MNRAS, 398, 898

Hubble, E. P. 1926, ApJ, 64, 321

Hubble, E. P. 1936, Realm of the Nebulae (New Haven: Yale University Press)

Huertas-Company, M., Pérez-González, P. G., Mei, S., et al. 2015, ApJ, 809, 95

Hunter, J. D. 2007, Comput. Sci. Eng., 9, 90

Ilbert, O., Arnouts, S., McCracken, H. J., et al. 2006, A\&A, 457, 841

Jones, E., Oliphant, T., Peterson, P., et al. 2001, SciPy: Open Source Scientific Tools for Python, http://www. scipy .org

Kaviraj, S., Devriendt, J., Dubois, Y., et al. 2015, MNRAS, 452, 2845

Kehrig, C., Monreal-Ibero, A., Papaderos, P., et al. 2012, A\&A, 540, A11

Koekemoer, A. M., Faber, S. M., Ferguson, H. C., et al. 2011, ApJS, 197, 36

Kormendy, J. 1977, ApJ, 218, 333

Kormendy, J., \& Ho, L. C. 2013, ARA\&A, 51, 511

Kormendy, J., \& Kennicutt, Jr., R. C. 2004, ARA\&A, 42, 603

Krist, J. E., Hook, R. N., \& Stoehr, F. 2011, in Optical Modeling and Performance Predictions V, Proc. SPIE, 8127, 81270J

Lackner, C. N., \& Gunn, J. E. 2012, MNRAS, 421, 2277

Lange, R., Moffett, A. J., Driver, S. P., et al. 2016, MNRAS, 462, 1470

López-Sanjuan, C., Balcells, M., Pérez-González, P. G., et al. 2010, ApJ, 710, 1170

López-Sanjuan, C., Le Fèvre, O., de Ravel, L., et al. 2011, A\&A, 530, A20

López-Sanjuan, C., Le Fèvre, O., Ilbert, O., et al. 2012, A\&A, 548, A7

Mármol-Queraltó, E., Trujillo, I., Pérez-González, P. G., Varela, J., \& Barro, G. 2012, MNRAS, 422, 2187

Meert, A., Vikram, V., \& Bernardi, M. 2015, MNRAS, 446, 3943

Mendel, J. T., Simard, L., Palmer, M., Ellison, S. L., \& Patton, D. R. 2014, ApJS, 210,3

Méndez-Abreu, J., Aguerri, J. A. L., Corsini, E. M., \& Simonneau, E. 2008, A\&A, 478, 353

Oke, J. B., \& Gunn, J. E. 1983, ApJ, 266, 713

Oser, L., Ostriker, J. P., Naab, T., Johansson, P. H., \& Burkert, A. 2010, ApJ, 725, 2312

Papaderos, P., Loose, H.-H., Thuan, T. X., \& Fricke, K. J. 1996, A\&AS, 120, 207

Peng, C. Y., Ho, L. C., Impey, C. D., \& Rix, H.-W. 2002, AJ, 124, 266

Peng, C. Y., Ho, L. C., Impey, C. D., \& Rix, H.-W. 2010, AJ, 139, 2097

Ribeiro, B., Lobo, C., Antón, S., Gomes, J. M., \& Papaderos, P. 2016, MNRAS, 456, 3899

Robitaille, T., \& Bressert, E. 2012, Astrophysics Source Code Library [record ascl:1208.017]

Sandin, C. 2014, A\&A, 567, A97

Sandin, C. 2015, A\&A, 577, A106

Scannapieco, C., Gadotti, D. A., Jonsson, P., \& White, S. D. M. 2010, MNRAS, 407, L41

Sérsic, J. L. 1963, Boletin de la Asociacion Argentina de Astronomia La Plata Argentina, 6, 41

Shen, S., Mo, H. J., White, S. D. M., et al. 2003, MNRAS, 343, 978

Shimasaku, K., Fukugita, M., Doi, M., et al. 2001, AJ, 122, 1238

Simard, L., Willmer, C. N. A., Vogt, N. P., et al. 2002, ApJS, 142, 1

Simard, L., Mendel, J. T., Patton, D. R., Ellison, S. L., \& McConnachie, A. W. 2011, ApJS, 196, 11

Simien, F., \& de Vaucouleurs, G. 1986, ApJ, 302, 564

Skelton, R. E., Whitaker, K. E., Momcheva, I. G., et al. 2014, ApJS, 214, 24

Taylor, M. B. 2005, in Astronomical Data Analysis Software and Systems XIV, eds. P. Shopbell, M. Britton, \& R. Ebert, ASP Conf. Ser., 347, 29

Trujillo, I., Förster Schreiber, N. M., Rudnick, G., et al. 2006, ApJ, 650, 18

Trujillo, I., Conselice, C. J., Bundy, K., et al. 2007, MNRAS, 382, 109

van der Wel, A., Bell, E. F., Häussler, B., et al. 2012, ApJS, 203, 24

van der Wel, A., Franx, M., van Dokkum, P. G., et al. 2014, ApJ, 788, 28

van Dokkum, P. G., Whitaker, K. E., Brammer, G., et al. 2010, ApJ, 709, 1018 


\section{Appendix A: Simulations to test the estimates of the structural parameters}

We conducted a set of simulations to test the robustness of our measured structural parameters for the $H$ and the $I$ bands. We used the ranges of the structural parameters from our analysis as a guide to create our artificial galaxies, uniformly randomized within the ranges $15.5<H_{\mathrm{AB}}[\mathrm{mag}]<19$, $0.3<r_{\mathrm{e}, H \text {-band }}[\operatorname{arcsec}]<3,1<n_{H \text {-band }}<6,0.4<a r<1$, where $H_{\mathrm{AB}}, r_{\mathrm{e}, H \text {-band }}, n_{H \text {-band }}$, and $a r$ stand for the derived $H_{\mathrm{AB}}$-band magnitude, effective radius, Sérsic index, and axis ratio, respectively. For the $I$ band we used the values of the structural parameters, which were uniformly randomized within the ranges $15.5<I_{\mathrm{AB}}[\mathrm{mag}]<20,0.3<r_{\mathrm{e}, I \text {-band }}[\operatorname{arcsec}]<3,1<n_{I \text {-band }}<7$, $0.4<a r<1$, where $I_{\mathrm{AB}}, r_{\mathrm{e}, I \text {-band }}, n_{I \text {-band }}$, and ar stand for the derived $I_{\mathrm{AB}}$-band magnitude, effective radius, Sérsic index, and axis ratio, respectively. Taking these values into account, we created 4500 mock galaxies for the $H$ band and 6000 for the $I$ band (because of the wider range of parameters for this band) uniformly distributed along the entire parameter space, placing each galaxy randomly on the corresponding band image and convolving with the respective PSF. We analyzed each artificial galaxy with the same methodology used in our real sample for the single Sérsic fits.

Figures A.1 and A.3 show the relative errors of the structural parameters calculated as (output-input)/input versus the input magnitude of the artificial galaxy. The sample is color-coded according to the input Sérsic index for the purpose of exploring the associated effects on the structural parameter. From these figures we can see that the recovery of the structural parameters in the $H$ band is more affected at increasing Sérsic index values. Low Sérsic index galaxies (blue points) are properly recovered even at the faintest magnitudes.

In Figs. A.2 and A.4 the sample is split into three groups according to their input magnitude to highlight the effect of the apparent magnitude in addition to the effect of the input Sérsic index (top panels) and the input effective radii (bottom panels). Fainter galaxies are more affected in recovering both the effective radius and the Sérsic index, independently of their input Sérsic index value.

Combining the results obtained with these simulations, we find that the apparent magnitude and the Sérsic index are the essential parameters for the good recovery of the structural parameters, and that the galaxy size plays a minor role.
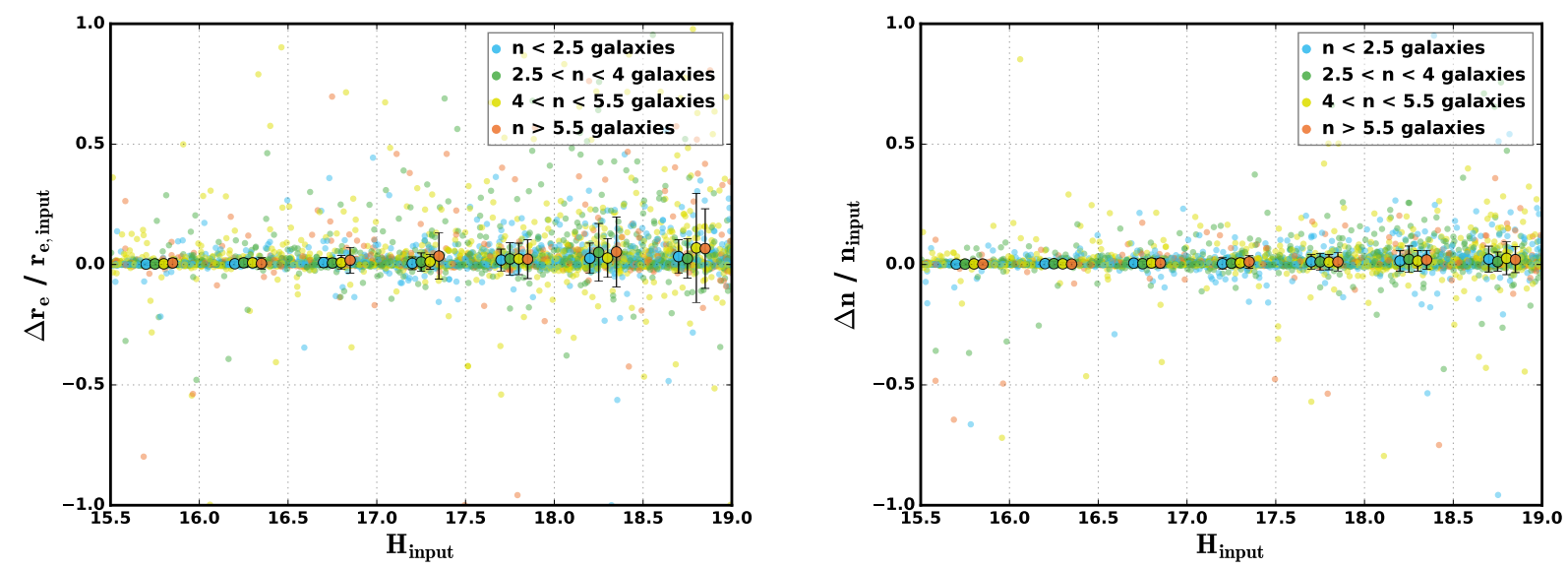

Fig. A.1. Relative errors - (output-input)/input - of the structural parameter effective radius (left panel) and Sérsic index (right panel) of our 4500 galaxies in the $H$ band. Galaxies are color-coded according to the input Sérsic index. The higher the input Sérsic index, the more affected the recovery of the structural parameters. 

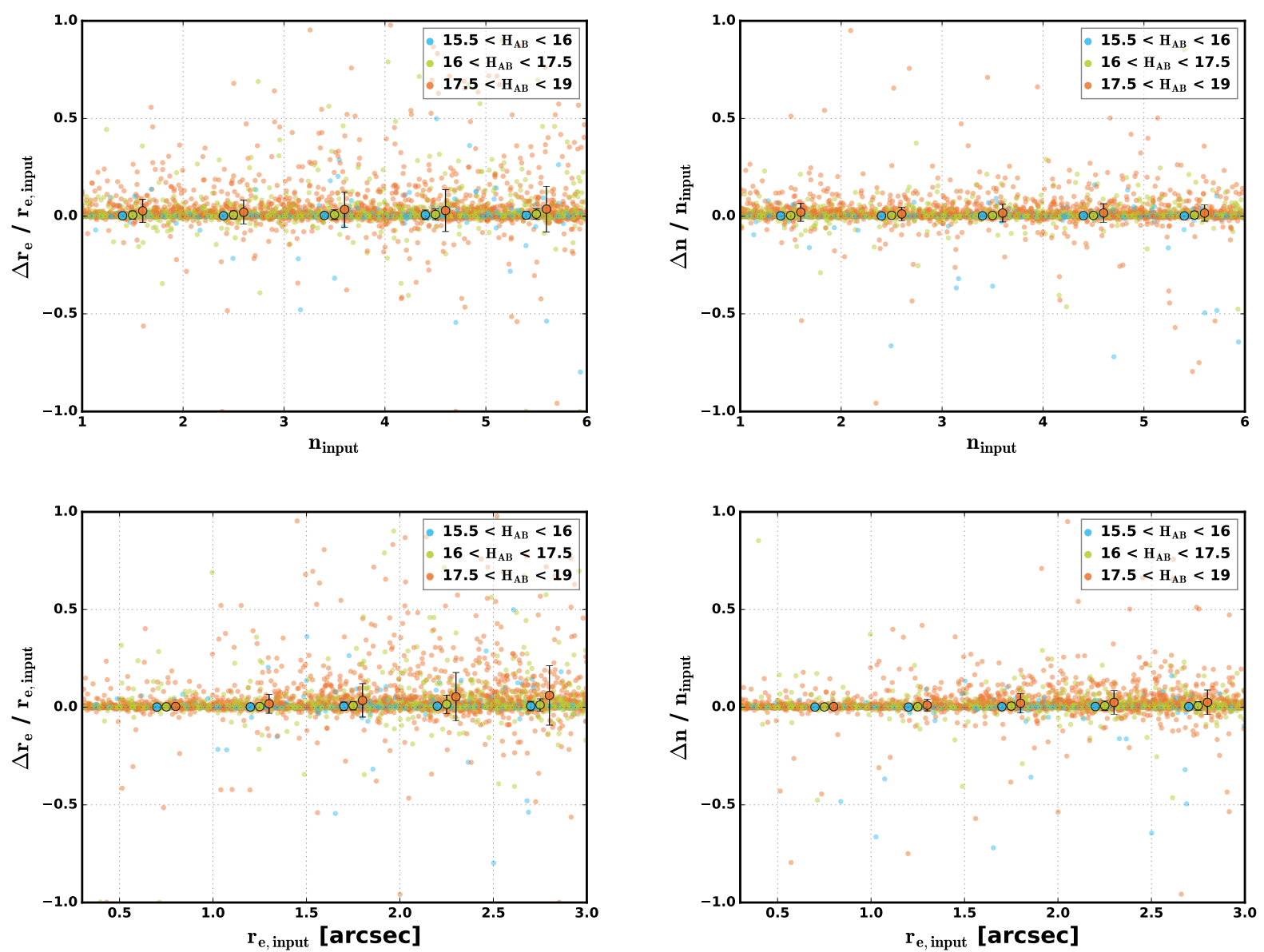

Fig. A.2. Relative errors - (output-input)/input - of the effective radius and the Sérsic index as a function of the input Sérsic index and the input effective radius in the $H$ band.
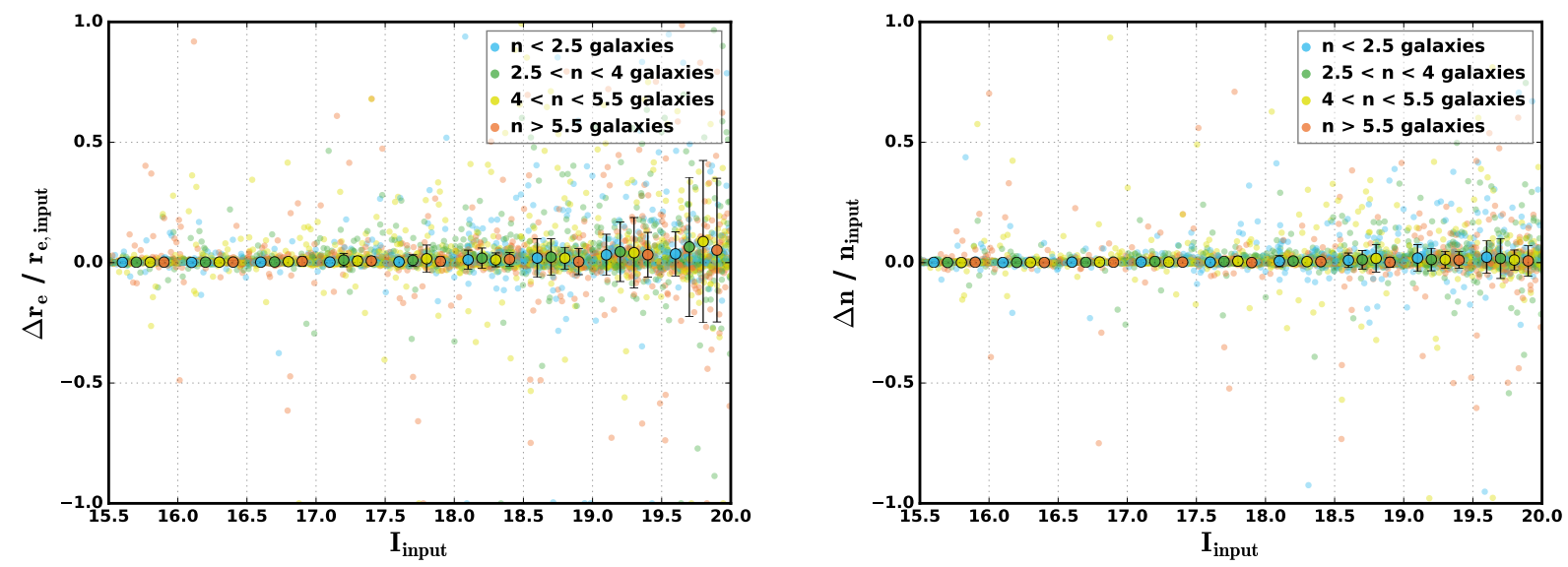

Fig. A.3. Relative errors - (output-input)/input - of the structural parameters of our 6000 galaxies in the $I$ band. Galaxies are color-coded according to the input Sérsic index. Fainter objects are more affected in the recovery of the structural parameters. 

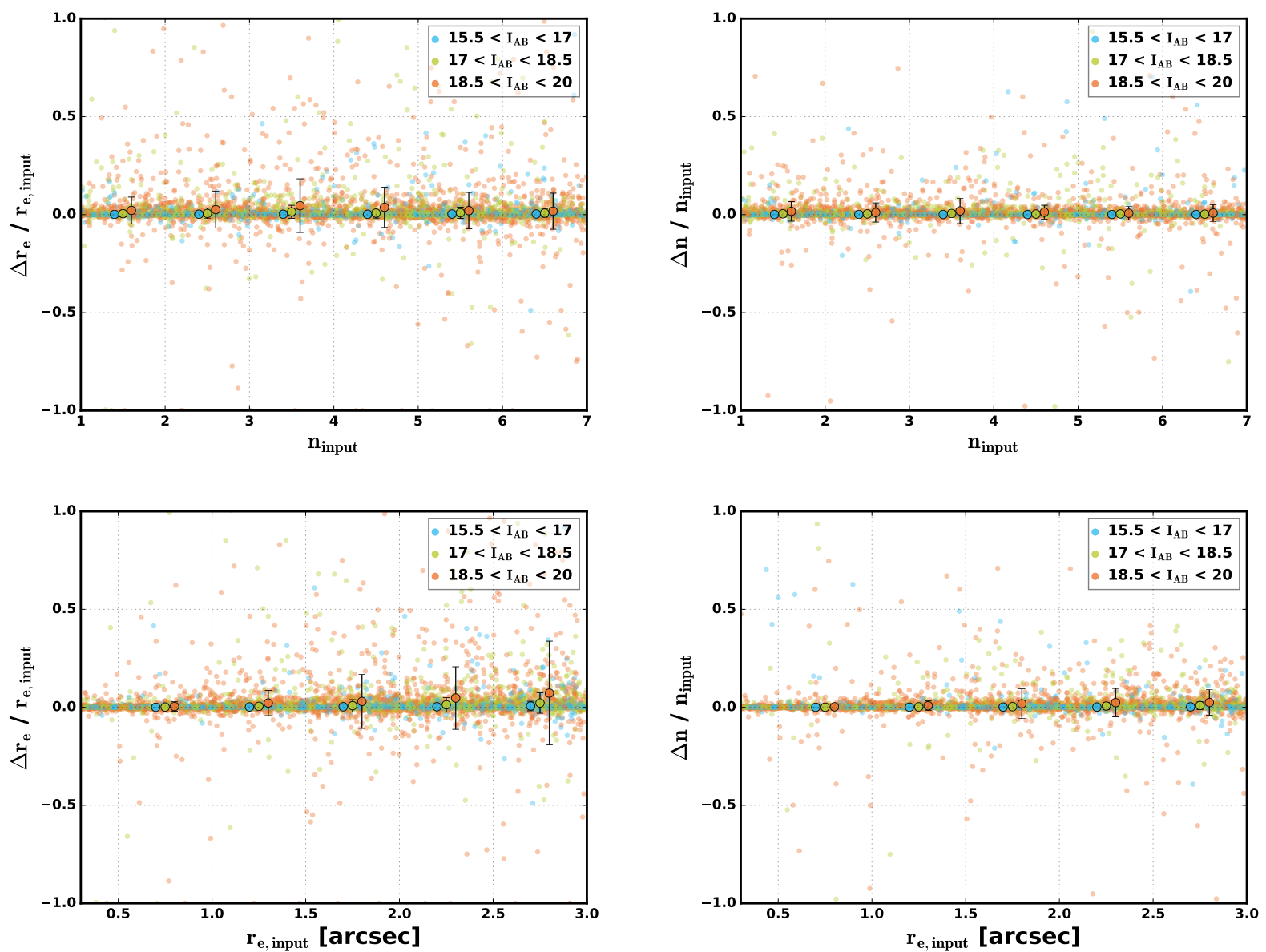

Fig. A.4. Relative errors - (output-input)/input - of the effective radius and the Sérsic index as a function of the input Sérsic index and the input effective radius in the $I$ band.

\section{Appendix B: Tables of the retrieved structural parameters}

Table B.1. Structural parameters for single Sérsic fits in the WFC3 $H$ band.

\begin{tabular}{lcccccccc}
\hline \hline Galaxy ID & mag $\pm \Delta$ mag & $\begin{array}{c}r_{\mathrm{e}} \pm \Delta r_{\mathrm{e}} \\
{[\mathrm{kpc}]}\end{array}$ & $n \pm \Delta n$ & $q$ & $\begin{array}{c}\text { pa } \\
{[\mathrm{deg}]}\end{array}$ & mag $_{\text {bar }}$ & $\begin{array}{c}r_{\mathrm{e}, \mathrm{bar}} \\
{[\mathrm{kpc}]}\end{array}$ & $\begin{array}{c}\mathrm{pa}_{\mathrm{bar}} \\
{[\mathrm{deg}]}\end{array}$ \\
\hline 37194 & $15.61 \pm 0.01$ & $2.63 \pm 0.05$ & $3.71 \pm 0.04$ & 0.57 & 66.17 & 15.95 & 5.35 & 60.22 \\
30654 & $17.98 \pm 0.01$ & $1.42 \pm 0.01$ & $2.80 \pm 0.02$ & 0.47 & -28.96 & - & - & - \\
37587 & $16.87 \pm 0.03$ & $9.30 \pm 0.41$ & $5.12 \pm 0.10$ & 0.65 & 18.64 & - & - & - \\
19195 & $17.40 \pm 0.05$ & $8.00 \pm 0.45$ & $3.97 \pm 0.09$ & 0.83 & 57.98 & - & - & - \\
3740 & $17.48 \pm 0.03$ & $3.45 \pm 0.14$ & $3.40 \pm 0.10$ & 0.68 & 64.87 & - & - & - \\
20050 & $17.54 \pm 0.03$ & $3.30 \pm 0.13$ & $3.85 \pm 0.12$ & 0.57 & -54.07 & - & - & - \\
10876 & $17.43 \pm 0.03$ & $3.88 \pm 0.15$ & $3.19 \pm 0.10$ & 0.96 & -87.80 & - & - & - \\
21796 & $17.50 \pm 0.03$ & $9.58 \pm 0.36$ & $5.29 \pm 0.17$ & 0.88 & 6.85 & - & - & - \\
18935 & $17.79 \pm 0.02$ & $9.03 \pm 0.19$ & $2.17 \pm 0.02$ & 0.40 & 86.65 & - & - & - \\
7013 & $18.17 \pm 0.01$ & $1.91 \pm 0.09$ & $2.73 \pm 0.03$ & 0.50 & -23.05 & - & - & - \\
21604 & $17.57 \pm 0.07$ & $8.40 \pm 0.47$ & $2.38 \pm 0.09$ & 0.63 & 59.67 & - & - & - \\
25781 & $17.77 \pm 0.06$ & $7.05 \pm 0.58$ & $3.99 \pm 0.23$ & 0.81 & -46.99 & - & - & - \\
1996 & $18.03 \pm 0.08$ & $4.70 \pm 0.51$ & $4.73 \pm 0.30$ & 0.71 & 75.63 & - & - & - \\
21306 & $18.66 \pm 0.03$ & $3.73 \pm 0.14$ & $3.67 \pm 0.17$ & 0.65 & 12.50 & - & - & - \\
23956 & $16.44 \pm 0.02$ & $6.28 \pm 0.22$ & $3.55 \pm 0.08$ & 0.41 & 47.39 & - & - & - \\
13942 & $18.41 \pm 0.28$ & $8.84 \pm 0.93$ & $1.47 \pm 0.23$ & 0.57 & -59.77 & 20.30 & 0.84 & 78.48 \\
4735 & $18.01 \pm 0.03$ & $3.79 \pm 0.15$ & $3.42 \pm 0.16$ & 0.59 & 55.30 & - & - & - \\
\hline
\end{tabular}

Notes. Column descriptions: (1) galaxy ID, (2) magnitude, (3) effective radius, (4) Sérsic index, (5) axis ratio, (6) position angle, (7) magnitude of the bar component, (8) effective radius of the bar component, (9) position angle of the bar component. 
In this appendix we present the results of the analysis of our sample with GALFIT. Tables B.1 and B.2 list the single Sérsic fits for the $H$ and $I$ band, respectively, containing the structural

parameters. Tables B.3 and B.4 are related to the multicomponent Sérsic fits in the $H$ and $I$ bands, respectively.

Table B.2. Structural parameters for single Sérsic fits in the ACS I band.

\begin{tabular}{lcccccccc}
\hline \hline Galaxy ID & mag $\pm \Delta$ mag & $\begin{array}{c}r_{\mathrm{e}} \pm \Delta r_{\mathrm{e}} \\
{[\mathrm{kpc}]}\end{array}$ & $n \pm \Delta n$ & $q$ & $\begin{array}{c}\text { pa } \\
{[\mathrm{deg}]}\end{array}$ & $\operatorname{mag}_{\mathrm{bar}}$ & $\begin{array}{c}r_{\mathrm{e}, \mathrm{bar}} \\
{[\mathrm{kpc}]}\end{array}$ & $\begin{array}{c}\mathrm{pa}_{\mathrm{bar}} \\
{[\mathrm{deg}]}\end{array}$ \\
\hline 37194 & $15.33 \pm 0.03$ & $9.26 \pm 0.56$ & $5.00 \pm 0.10$ & 0.65 & 63.89 & 19.22 & 4.63 & 69.33 \\
30654 & $18.87 \pm 0.01$ & $1.54 \pm 0.02$ & $3.20 \pm 0.26$ & 0.49 & -27.65 & - & - & - \\
37587 & $17.97 \pm 0.02$ & $9.13 \pm 0.25$ & $4.45 \pm 0.05$ & 0.58 & 15.81 & - & - & - \\
19195 & $18.50 \pm 0.02$ & $6.82 \pm 0.19$ & $3.37 \pm 0.04$ & 0.85 & 48.89 & - & - & - \\
3740 & $18.31 \pm 0.01$ & $4.71 \pm 0.07$ & $4.04 \pm 0.03$ & 0.67 & 64.78 & - & - & - \\
20050 & $18.52 \pm 0.02$ & $6.20 \pm 0.18$ & $4.47 \pm 0.04$ & 0.62 & -47.46 & - & - & - \\
10876 & $18.38 \pm 0.04$ & $4.52 \pm 0.43$ & $3.38 \pm 0.26$ & 0.97 & -30.69 & - & - & - \\
21796 & $19.06 \pm 0.03$ & $4.95 \pm 0.20$ & $4.20 \pm 0.08$ & 0.80 & -0.79 & - & - & - \\
18935 & $18.74 \pm 0.06$ & $12.06 \pm 0.89$ & $2.35 \pm 0.09$ & 0.46 & 84.31 & - & - & - \\
7013 & $19.40 \pm 0.01$ & $2.34 \pm 0.04$ & $2.71 \pm 0.02$ & 0.49 & -20.02 & - & - & - \\
25781 & $18.63 \pm 0.07$ & $11.93 \pm 1.27$ & $4.97 \pm 0.20$ & 0.77 & -37.28 & - & - & - \\
1996 & $19.11 \pm 0.08$ & $5.59 \pm 0.53$ & $5.01 \pm 0.20$ & 0.76 & 78.80 & - & - & - \\
21306 & $19.79 \pm 0.21$ & $3.81 \pm 0.67$ & $3.78 \pm 0.47$ & 0.62 & 9.31 & - & - & - \\
23956 & $17.19 \pm 0.09$ & $8.33 \pm 0.92$ & $4.20 \pm 0.26$ & 0.45 & 46.33 & - & - & - \\
13942 & $19.02 \pm 0.05$ & $13.27 \pm 1.11$ & $5.27 \pm 0.16$ & 0.75 & -79.53 & 22.82 & 4.40 & -50.36 \\
4735 & $19.02 \pm 0.03$ & $4.88 \pm 0.20$ & $4.15 \pm 0.08$ & 0.64 & 53.86 & - & - & - \\
\hline
\end{tabular}

Notes. Column descriptions: (1) galaxy ID, (2) magnitude, (3) effective radius, (4) Sérsic index, (5) axis ratio, (6) position angle, (7) magnitude of the bar component, (8) effective radius of the bar component, (9) position angle of the bar component.

Table B.3. Structural parameters for multi-component Sérsic fits in the WFC3 $H$ band.

\begin{tabular}{|c|c|c|c|c|c|c|c|c|c|c|c|c|c|}
\hline Galaxy ID & $\operatorname{mag}_{\text {disk }}$ & $\begin{array}{l}r_{\mathrm{e}, \mathrm{disk}} \\
{[\mathrm{kpc}]}\end{array}$ & $n_{\text {disk }}$ & $q_{\text {disk }}$ & $\begin{array}{c}\mathrm{pa}_{\text {disk }} \\
\text { [degs] }\end{array}$ & mag $_{\text {bulge }}$ & $\begin{array}{c}r_{\mathrm{e}, \text { bulge }} \\
{[\mathrm{kpc}]}\end{array}$ & $n_{\text {bulge }}$ & $q_{\text {bulge }}$ & $\begin{array}{c}\text { pa }_{\text {bulge }} \\
\text { [deg] }\end{array}$ & $\operatorname{mag}_{\text {bar }}$ & $\begin{array}{c}r_{\mathrm{e}, \mathrm{bar}} \\
{[\mathrm{kpc}]}\end{array}$ & $\begin{array}{l}\mathrm{pa}_{\mathrm{bar}} \\
\text { [deg] }\end{array}$ \\
\hline 37194 & 15.17 & 5.42 & 1 & 0.54 & 64.95 & 18.10 & 0.23 & 1.21 & 0.70 & 66.25 & 17.48 & 0.82 & 64.39 \\
\hline 30654 & 18.51 & 2.62 & 1 & 0.30 & -25.64 & 19.16 & 0.39 & 0.94 & 0.79 & -51.10 & - & - & - \\
\hline 37587 & 17.81 & 8.20 & 1 & 0.50 & 16.19 & 18.36 & 1.46 & 2.58 & 0.72 & 21.95 & - & - & - \\
\hline 19195 & 19.72 & 1.01 & 1 & 0.77 & 39.61 & 17.57 & 9.49 & 2.26 & 0.83 & 67.56 & - & - & - \\
\hline 3740 & 19.60 & 0.65 & 1 & 0.70 & 79.64 & 17.58 & 4.82 & 2.35 & 0.66 & 60.08 & - & - & - \\
\hline 20050 & 19.74 & 0.74 & 1 & 0.28 & -52.47 & 17.53 & 5.50 & 3.79 & 0.60 & -51.87 & - & - & - \\
\hline 10876 & 20.11 & 0.57 & 1 & 0.78 & 54.67 & 17.61 & 4.01 & 2.07 & 0.98 & -67.44 & - & - & - \\
\hline 21796 & 18.27 & 9.90 & 1 & 0.93 & 63.25 & 18.88 & 1.64 & 2.30 & 0.82 & 1.39 & - & - & - \\
\hline 18935 & 18.05 & 8.60 & 1 & 0.35 & 86.25 & 20.64 & 0.92 & 1.06 & 0.74 & 84.02 & - & - & - \\
\hline 7013 & 19.20 & 6.52 & 1 & 0.22 & -20.12 & 18.70 & 0.99 & 1.67 & 0.64 & -26.39 & - & - & - \\
\hline 21604 & 17.96 & 8.03 & 1 & 0.67 & 73.91 & 19.45 & 2.86 & 2.55 & 0.39 & 46.43 & - & - & - \\
\hline 25781 & 20.31 & 0.66 & 1 & 0.86 & -49.91 & 18.14 & 5.94 & 1.77 & 0.80 & -44.76 & - & - & - \\
\hline 1996 & 20.32 & 0.46 & 1 & 0.85 & 82.31 & 18.20 & 5.82 & 3.00 & 0.71 & 70.76 & - & - & - \\
\hline 21306 & 19.71 & 1.06 & 1 & 0.67 & 9.04 & 19.35 & 6.26 & 0.61 & 0.71 & 23.99 & - & - & - \\
\hline 23956 & 16.94 & 6.71 & 1 & 0.31 & 46.11 & 18.32 & 0.76 & 1.37 & 0.78 & 70.39 & - & - & - \\
\hline 13942 & 18.65 & 10.29 & 1 & 0.77 & -79.67 & 20.16 & 6.35 & 0.35 & 0.27 & -53.23 & 20.05 & 0.93 & 83.19 \\
\hline 4735 & 20.42 & 0.64 & 1 & 0.53 & 46.22 & 18.12 & 4.77 & 2.66 & 0.57 & 55.32 & - & - & - \\
\hline
\end{tabular}

Notes. Column descriptions: (1) galaxy ID, (2) magnitude of the disk component, (3) effective radius of the disk component, (4) exponential Sérsic index, (5) axis ratio of the disk component, (6) position angle of the disk component, (7) magnitude of the bulge component, (8) effective radius of the bulge component, (9) Sérsic index of the bulge component, (10) axis ratio of the bulge component, (11) position angle of the bulge component, (12) magnitude of the bar component, (13) effective radius of the bar component, (14) position angle of the bar component. 
A\&A 634, A11 (2020)

Table B.4. Structural parameters for multi-component Sérsic fits in the ACS I band.

\begin{tabular}{|c|c|c|c|c|c|c|c|c|c|c|c|c|c|}
\hline Galaxy ID & $\operatorname{mag}_{\text {disk }}$ & $\begin{array}{l}r_{\mathrm{e}, \mathrm{disk}} \\
{[\mathrm{kpc}]}\end{array}$ & $n_{\text {disk }}$ & $q_{\text {disk }}$ & $\begin{array}{l}\text { pa }_{\text {disk }} \\
\text { [deg] }\end{array}$ & mag $_{\text {bulge }}$ & $\begin{array}{l}r_{\mathrm{e}, \text { bulge }} \\
{[\mathrm{kpc}]}\end{array}$ & $n_{\text {bulge }}$ & $q_{\text {bulge }}$ & $\begin{array}{c}\text { pa } a_{\text {bulge }} \\
{[\mathrm{deg}]}\end{array}$ & $\operatorname{mag}_{\text {bar }}$ & $\begin{array}{c}r_{\mathrm{e}, \mathrm{bar}} \\
{[\mathrm{kpc}]}\end{array}$ & $\begin{array}{c}\mathrm{pa}_{\mathrm{e}, \mathrm{bar}} \\
{[\mathrm{deg}]}\end{array}$ \\
\hline 37194 & 15.84 & 5.80 & 1 & 0.55 & 64.18 & 18.89 & 0.26 & 1.57 & 0.74 & 64.22 & 18.35 & 0.81 & 64.92 \\
\hline 30654 & 19.50 & 2.86 & 1 & 0.27 & -25.31 & 20.01 & 0.42 & 1.55 & 0.71 & -34.45 & - & - & - \\
\hline 37587 & 18.62 & 7.83 & 1 & 0.51 & 16.62 & 19.89 & 1.03 & 1.45 & 0.61 & 15.29 & - & - & - \\
\hline 19195 & 20.73 & 1.06 & 1 & 0.80 & 28.58 & 18.55 & 9.75 & 2.12 & 0.83 & 66.62 & - & - & - \\
\hline 3740 & 20.98 & 0.51 & 1 & 0.54 & 77.33 & 18.48 & 4.83 & 2.29 & 0.67 & 56.20 & - & - & - \\
\hline 20050 & 22.48 & 0.50 & 1 & 0.22 & -53.12 & 18.54 & 6.53 & 3.99 & 0.63 & -47.79 & - & - & - \\
\hline 10876 & 22.43 & 0.42 & 1 & 0.62 & -73.94 & 18.49 & 4.14 & 2.67 & 0.96 & -13.19 & - & - & - \\
\hline 21796 & 19.26 & 11.82 & 1 & 0.93 & 46.48 & 19.65 & 2.62 & 3.61 & 0.77 & -0.94 & - & - & - \\
\hline 18935 & 19.09 & 9.59 & 1 & 0.40 & 84.67 & 22.02 & 0.95 & 1.24 & 0.70 & 78.89 & - & - & - \\
\hline 7013 & 20.37 & 8.24 & 1 & 0.22 & -21.88 & 19.93 & 1.27 & 1.84 & 0.56 & -18.78 & - & - & - \\
\hline 25781 & 21.84 & 0.56 & 1 & 0.71 & -30.33 & 19.08 & 7.12 & 2.17 & 0.78 & -40.23 & - & - & - \\
\hline 1996 & 21.88 & 0.53 & 1 & 0.58 & -75.26 & 19.16 & 7.10 & 3.78 & 0.72 & 67.15 & - & - & - \\
\hline 21306 & 21.50 & 0.70 & 1 & 0.69 & 4.05 & 20.10 & 5.44 & 1.76 & 0.58 & 13.63 & - & - & - \\
\hline 23956 & 18.24 & 7.79 & 1 & 0.22 & 45.69 & 18.26 & 2.91 & 4.47 & 0.71 & 48.32 & - & - & - \\
\hline 13942 & 19.66 & 10.88 & 1 & 0.77 & -77.98 & 21.37 & 0.89 & 0.95 & 0.68 & 70.48 & 21.30 & 6.20 & -51.69 \\
\hline 4735 & 21.61 & 0.44 & 1 & 0.91 & 60.45 & 19.28 & 4.86 & 2.32 & 0.59 & 53.99 & - & - & - \\
\hline
\end{tabular}

Notes. Column descriptions: (1) galaxy ID, (2) magnitude of the disk component, (3) effective radius of the disk component, (4) exponential Sérsic index, (5) axis ratio of the disk component, (6) position angle of the disk component, (7) magnitude of the bulge component, (8) effective radius of the bulge component, (9) Sérsic index of the bulge component, (10) axis ratio of the bulge component, (11) position angle of the bulge component, (12) magnitude of the bar component, (13) effective radius of the bar component, (14) position angle of the bar component.

\section{Appendix C: Surface brightness profiles}
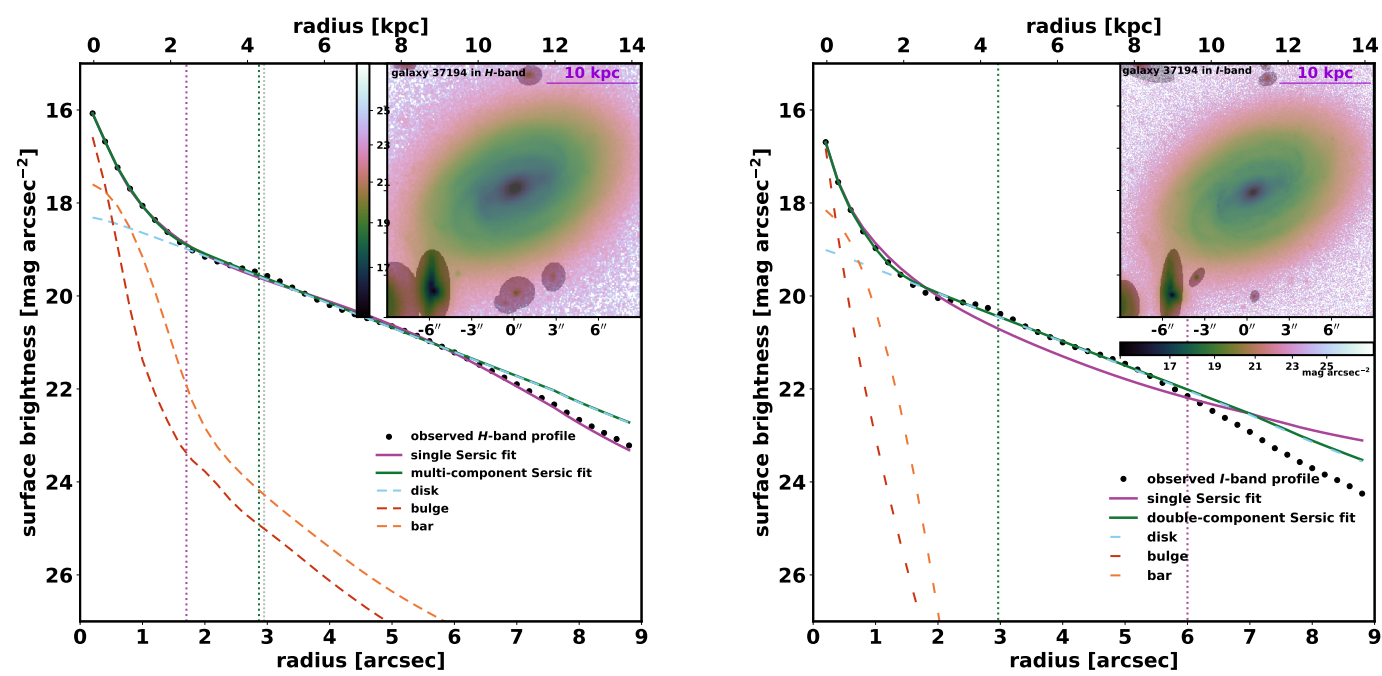

Fig. C.1. Surface brightness profiles of barred late-type galaxy 37194 in the $H$ band (left panel) and $I$ band (right panel). 

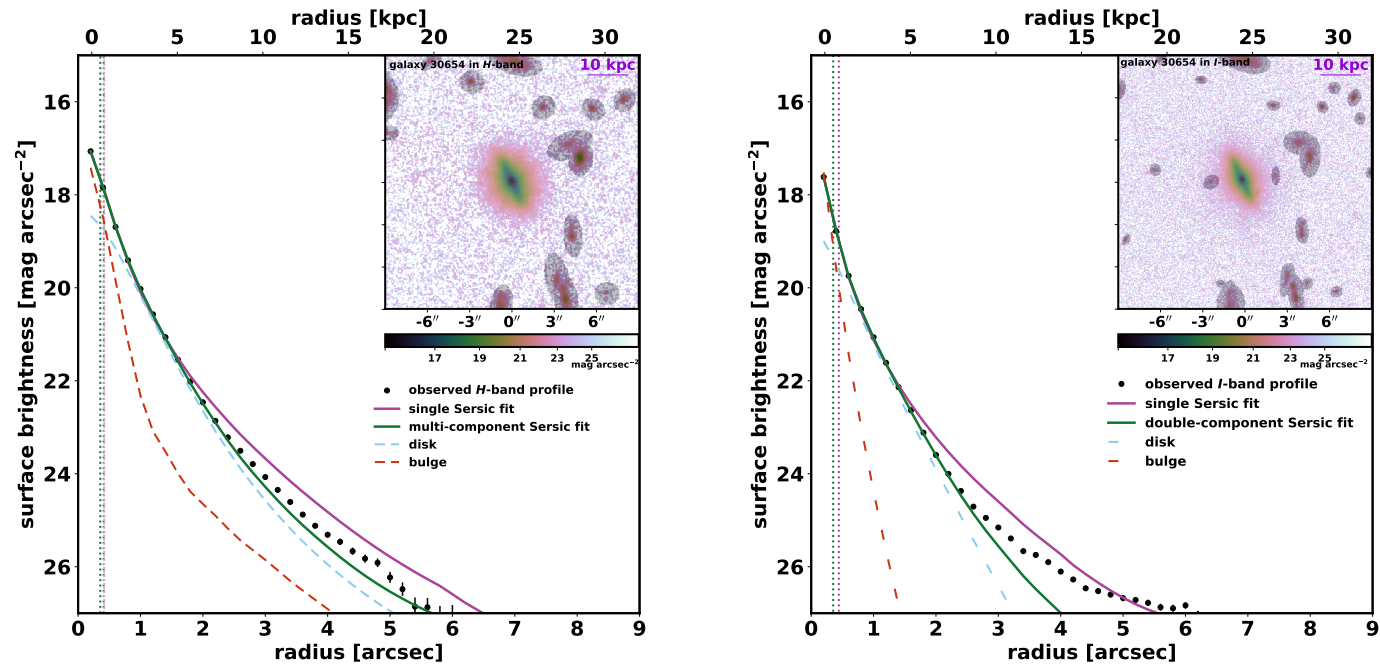

Fig. C.2. Surface brightness profiles of late-type galaxy 30654 in the $H$ band (left panel) and $I$ band (right panel).
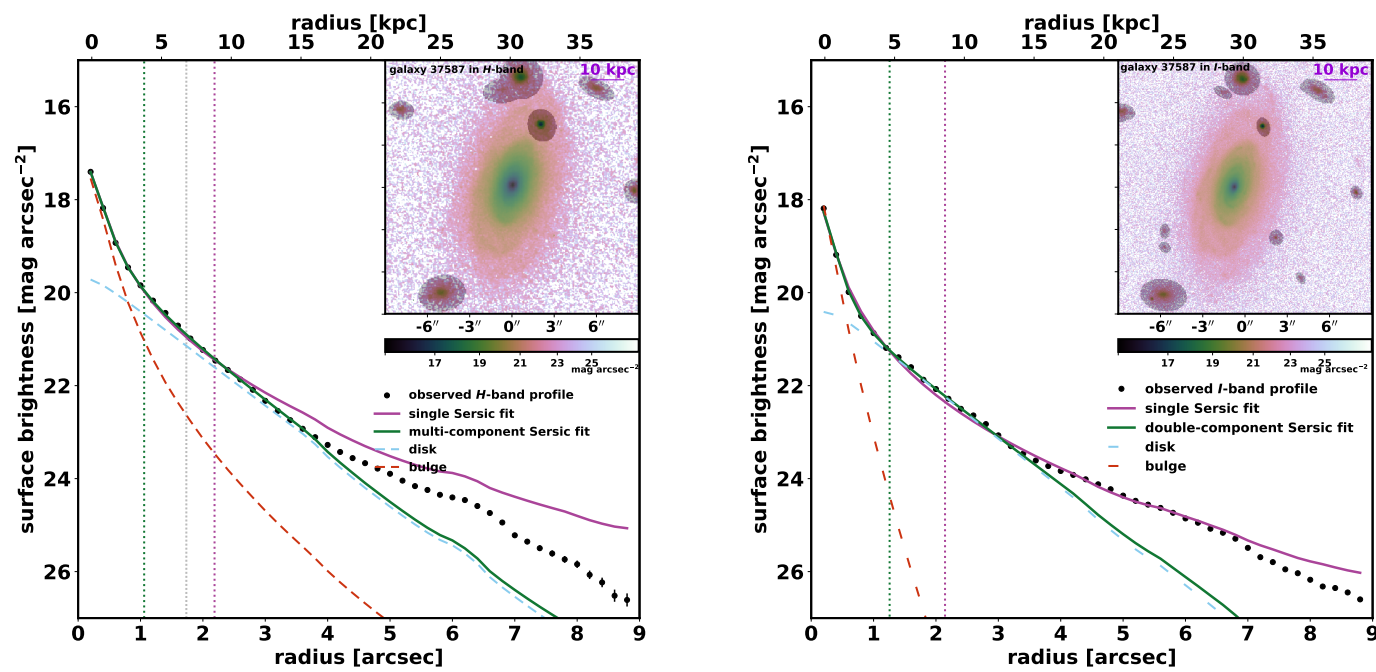

Fig. C.3. Surface brightness profiles of late-type galaxy 37587 in the $H$ band (left panel) and $I$ band (right panel).
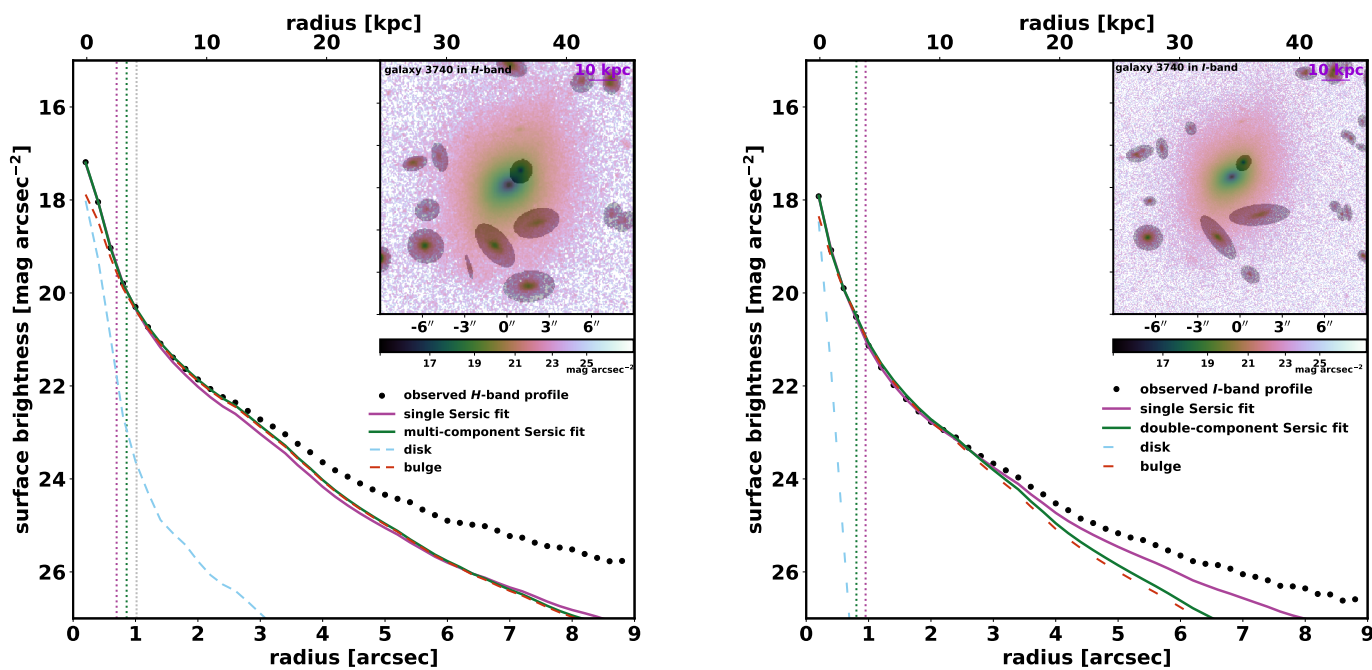

Fig. C.4. Surface brightness profiles of early-type galaxy 3740 in the $H$ band (left panel) and $I$ band (right panel). 

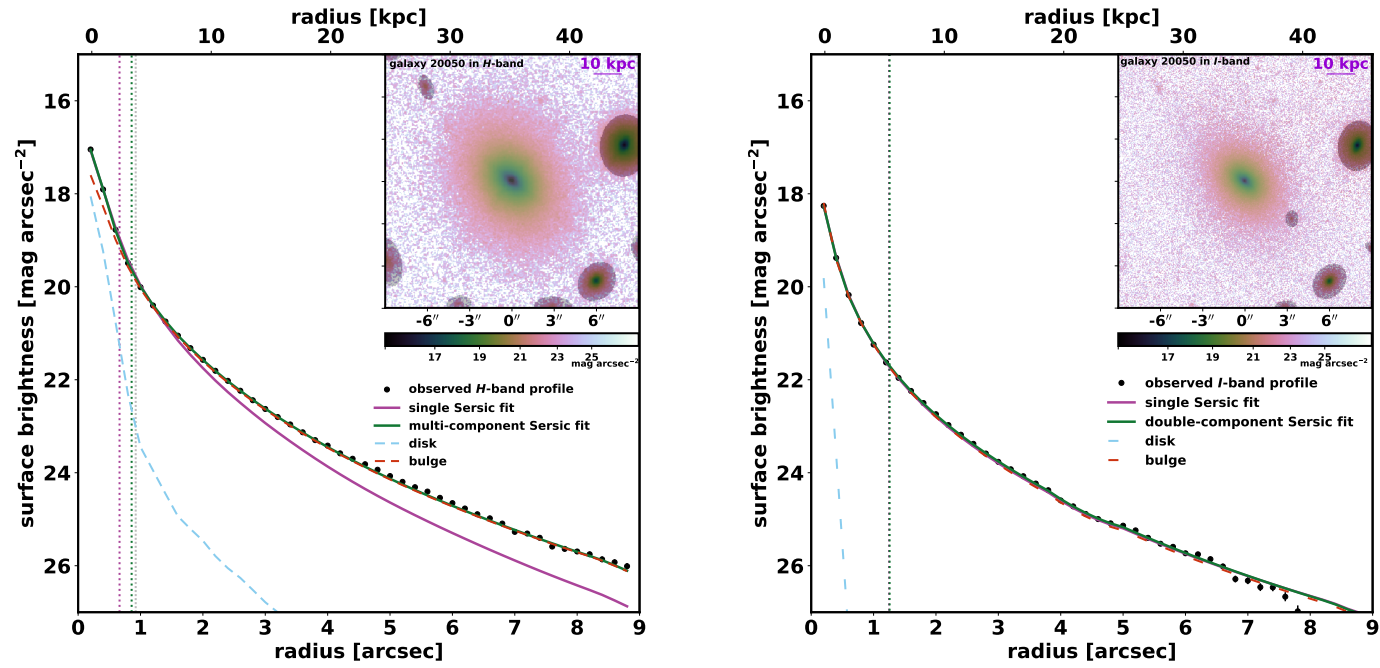

Fig. C.5. Surface brightness profiles of early-type galaxy 20050 in the $H$ band (left panel) and $I$ band (right panel).
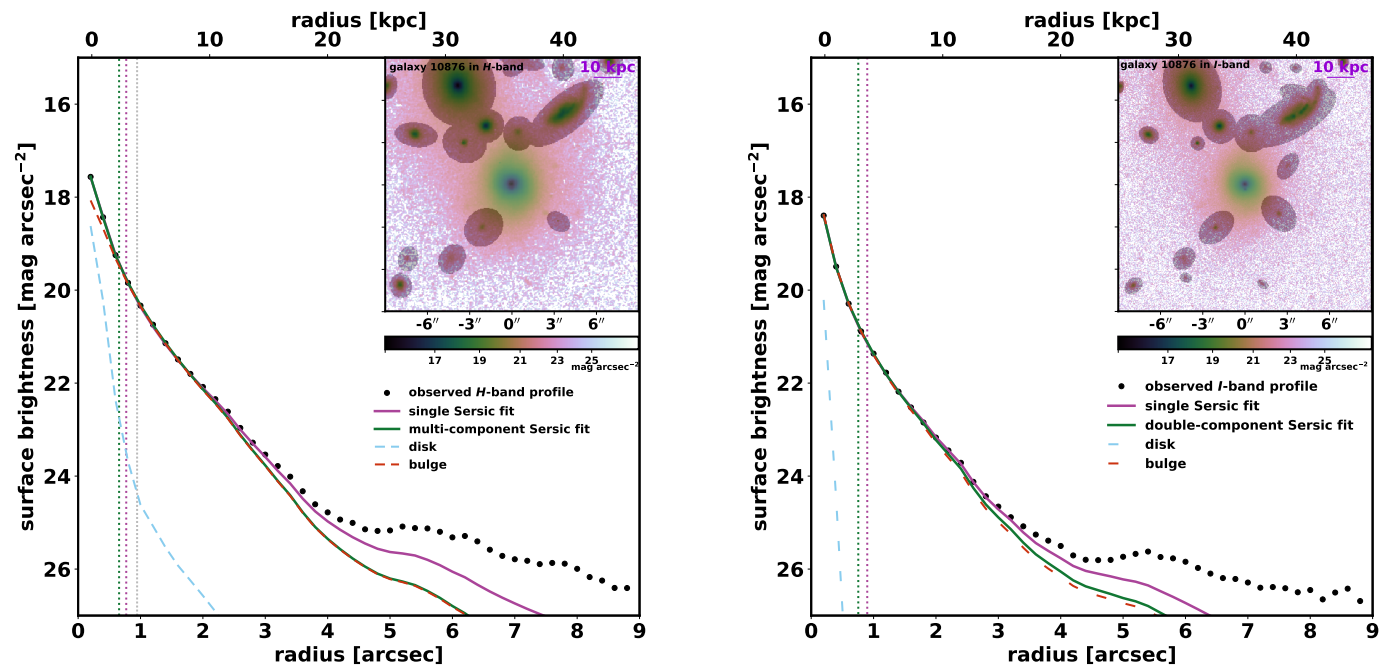

Fig. C.6. Surface brightness profiles of early-type galaxy 10876 in the $H$ band (left panel) and $I$ band (right panel).
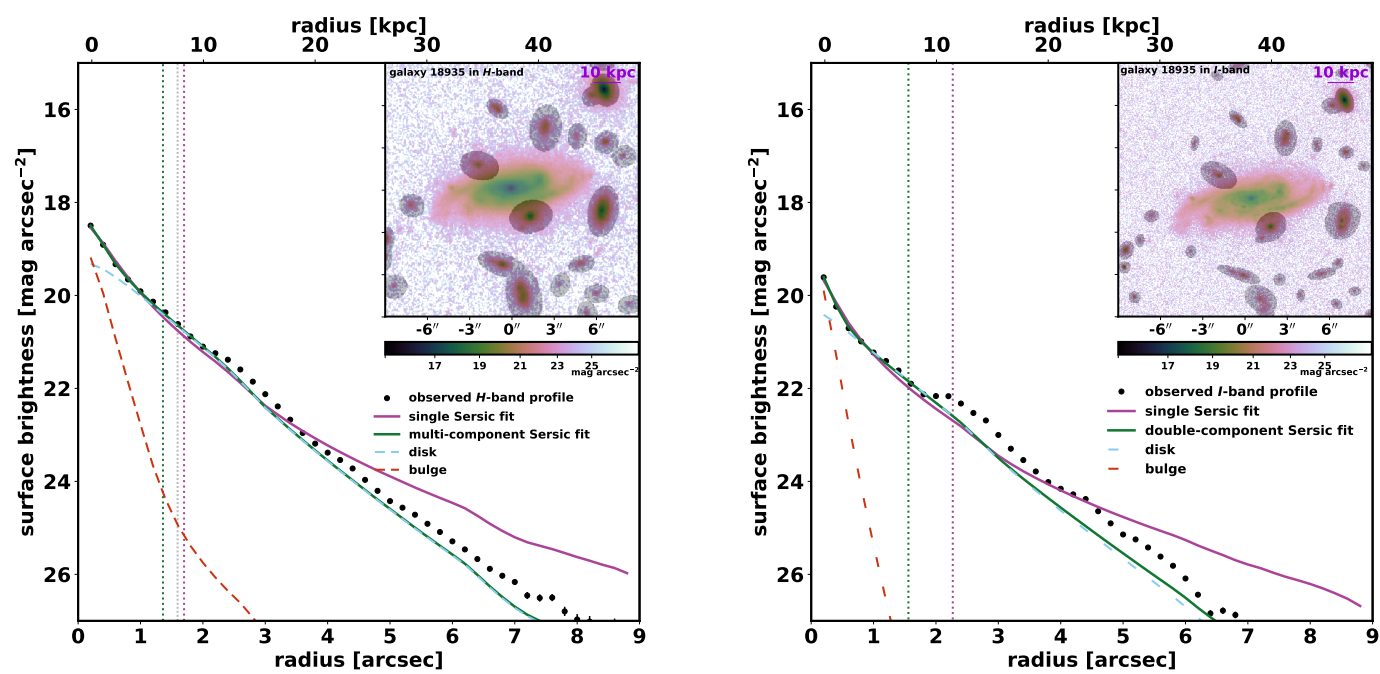

Fig. C.7. Surface brightness profiles of late-type galaxy 18935 in the $H$ band (left panel) and $I$ band (right panel). 


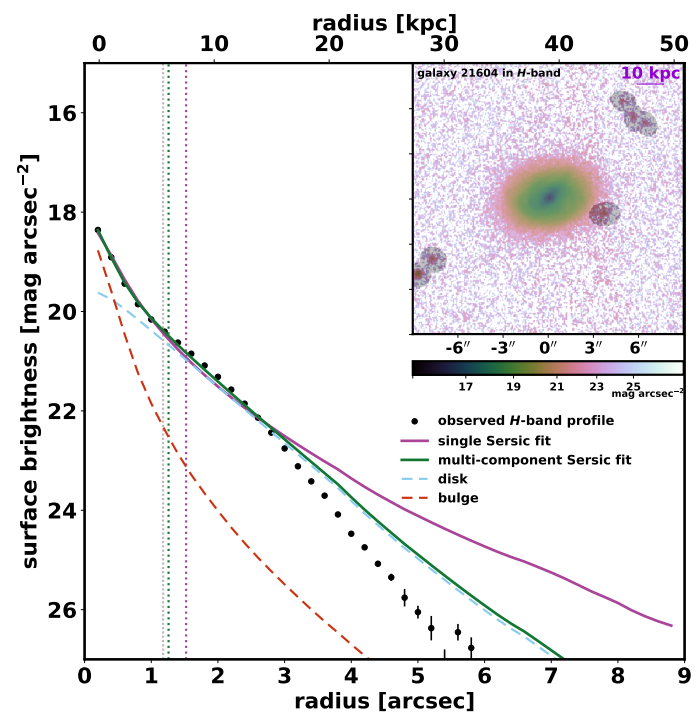

Fig. C.8. Surface brightness profiles of late-type galaxy 21604 in the $H$ band.
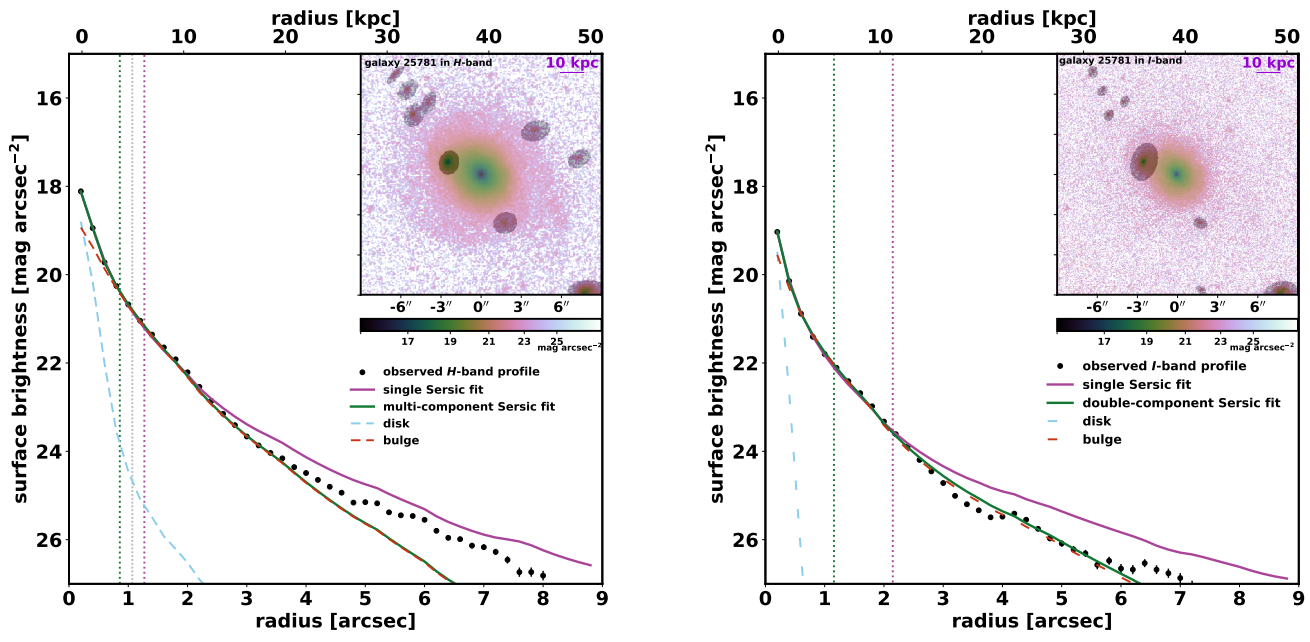

Fig. C.9. Surface brightness profiles of early-type galaxy 25781 in the $H$ band (left panel) and $I$ band (right panel).
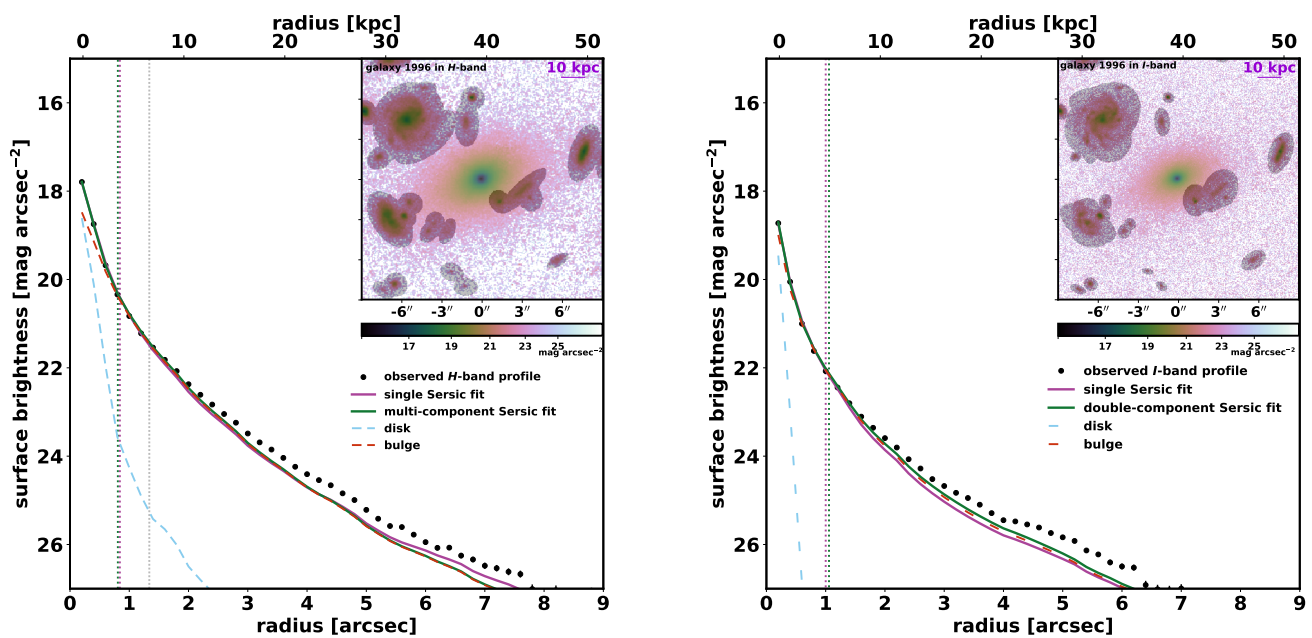

Fig. C.10. Surface brightness profiles of early-type galaxy 1996 in the $H$ band (left panel) and $I$ band (right panel). 

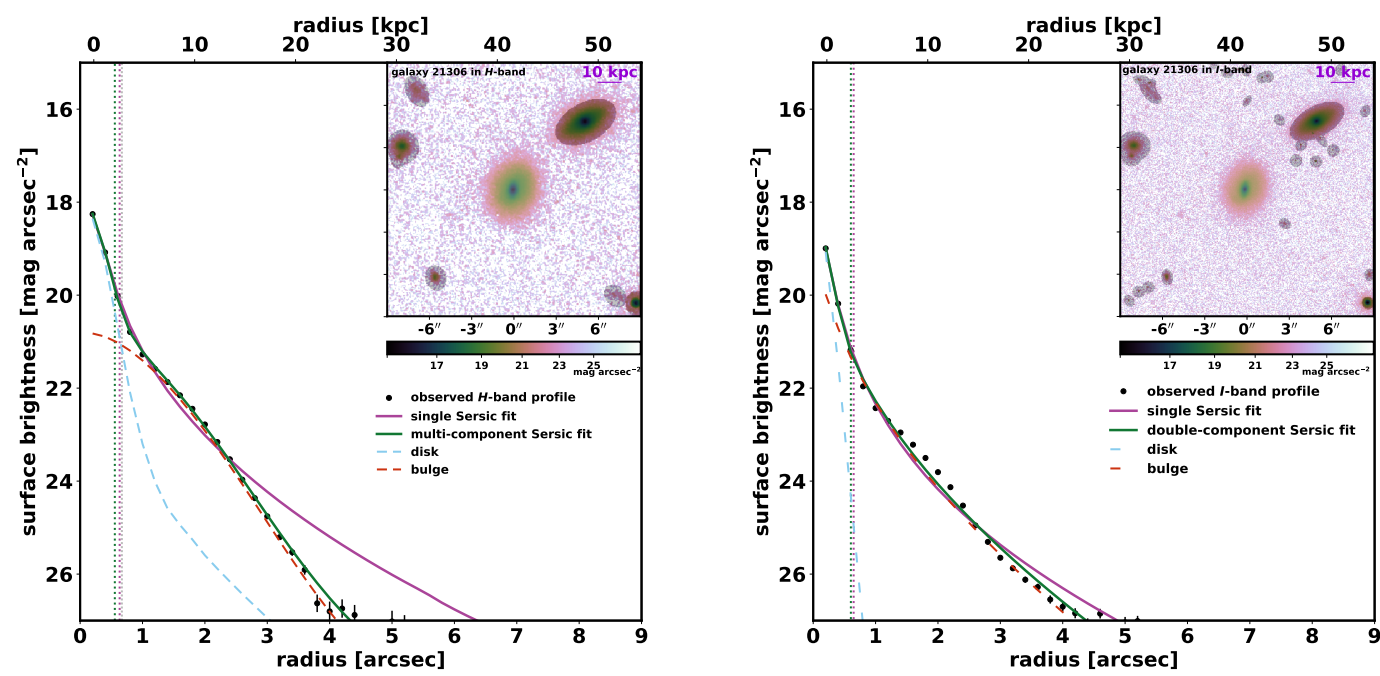

Fig. C.11. Surface brightness profiles of early-type galaxy 21306 in the $H$ band (left panel) and $I$ band (right panel).
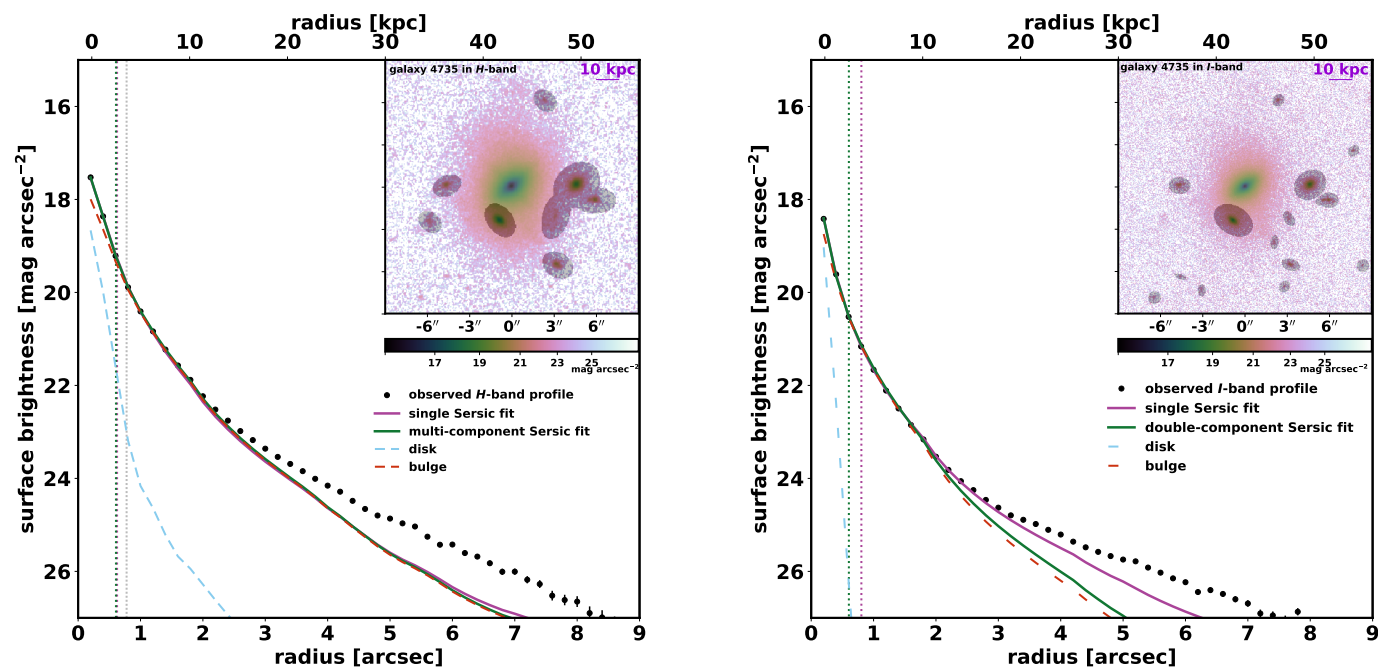

Fig. C.12. Surface brightness profiles of early-type galaxy 4735 in the $H$ band (left panel) and $I$ band (right panel).
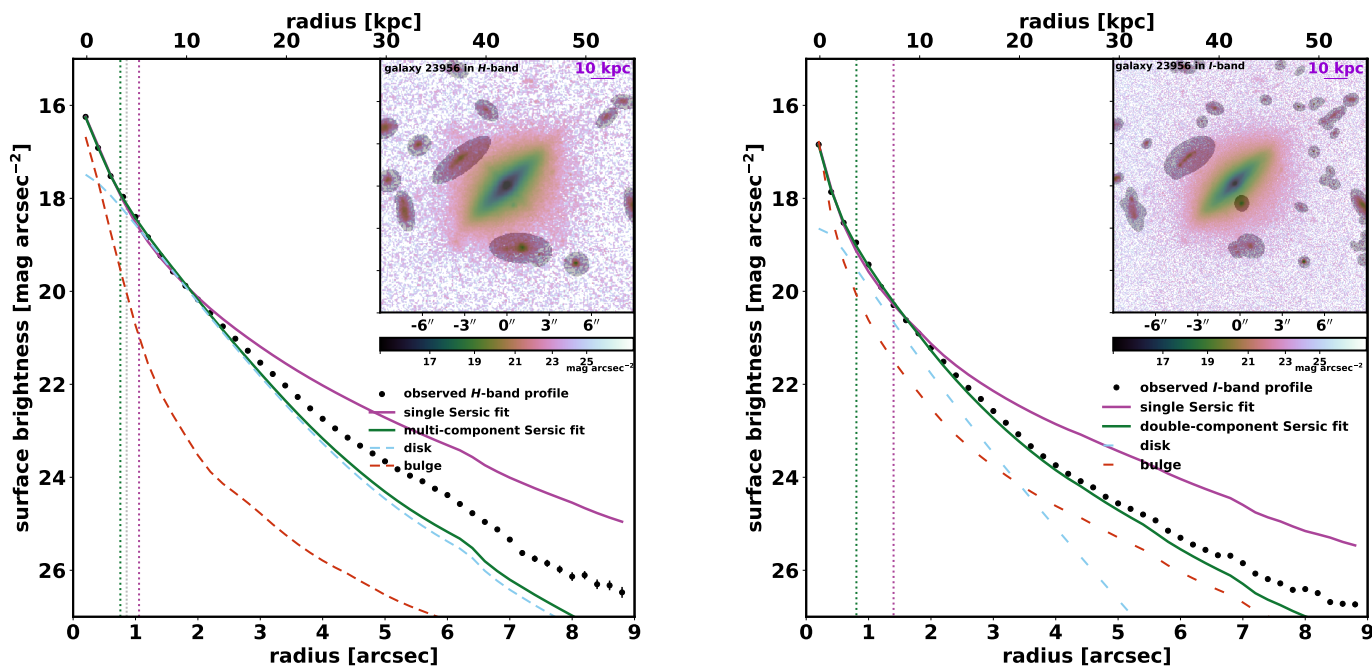

Fig. C.13. Surface brightness profiles of late-type galaxy 23956 in the $H$ band (left panel) and $I$ band (right panel). 
S. N. dos Reis et al.: Structural analysis of massive galaxies at $z<0.5$
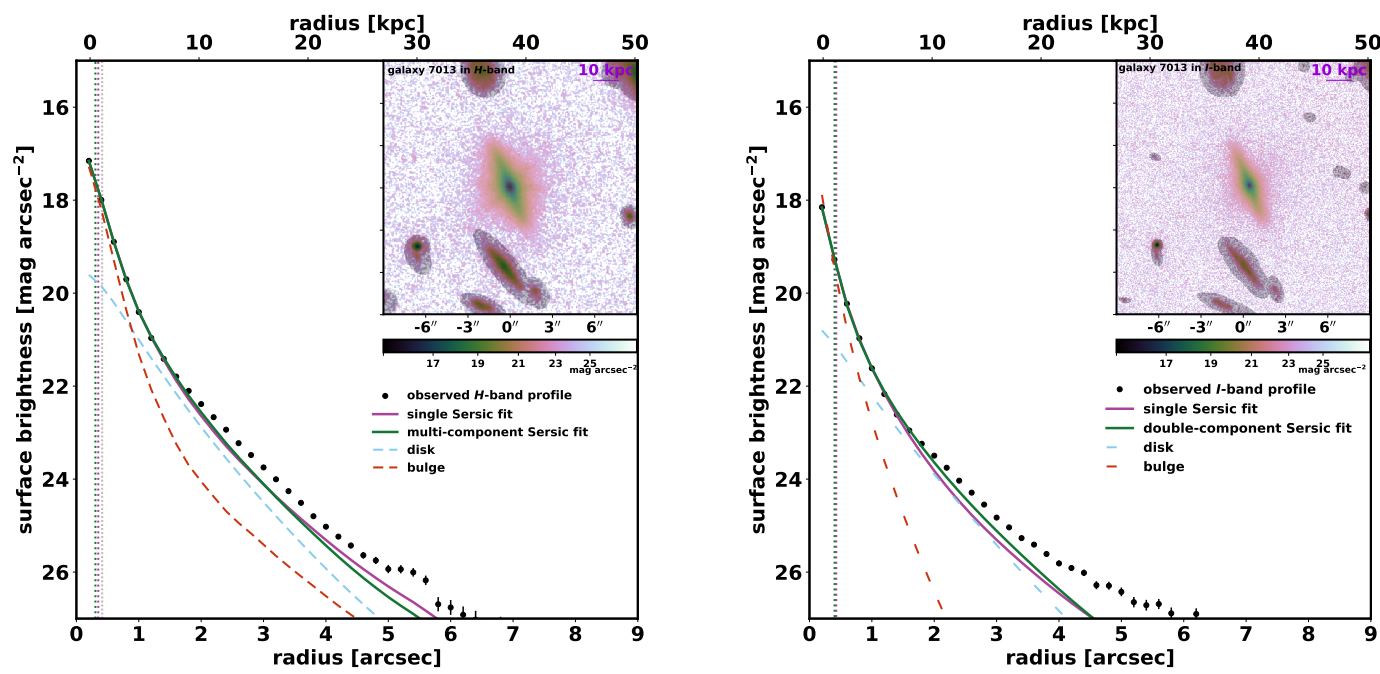

Fig. C.14. Surface brightness profiles of late-type galaxy 7013 in the $H$ band (left panel) and $I$ band (right panel). 


\section{Appendix D: Color profiles}
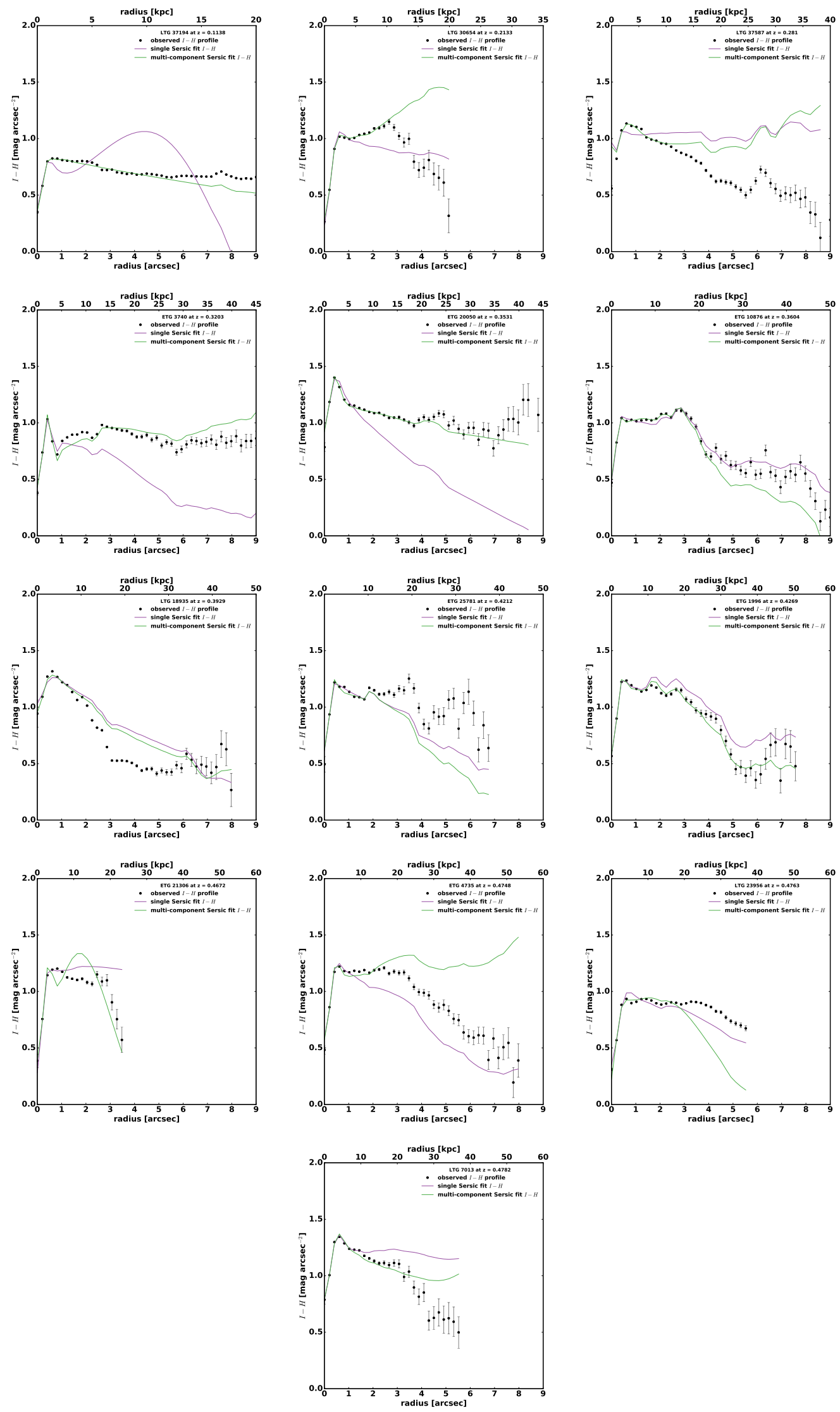

Fig. D.1. Color profiles for our sample. Data points with larger error bars $(>0.3 \mathrm{mag})$ are not shown. 


\section{Appendix E: Model residuals}

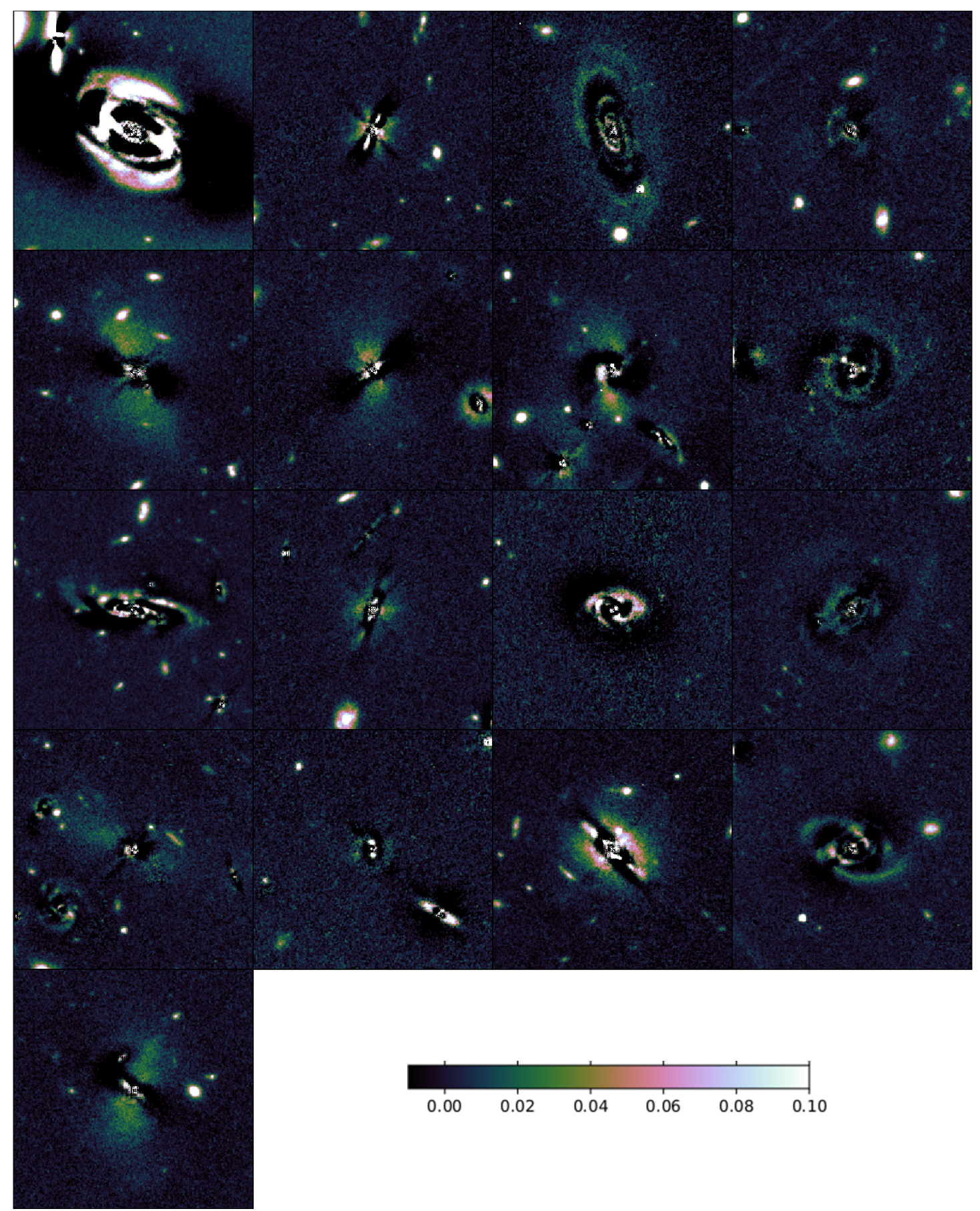

Fig. E.1. $H$-band residuals for the multi-component fits, after removal of the $\mathrm{B}+\mathrm{D}$ model from the original image. The images are in units of counts $\mathrm{s}^{-1}$. 


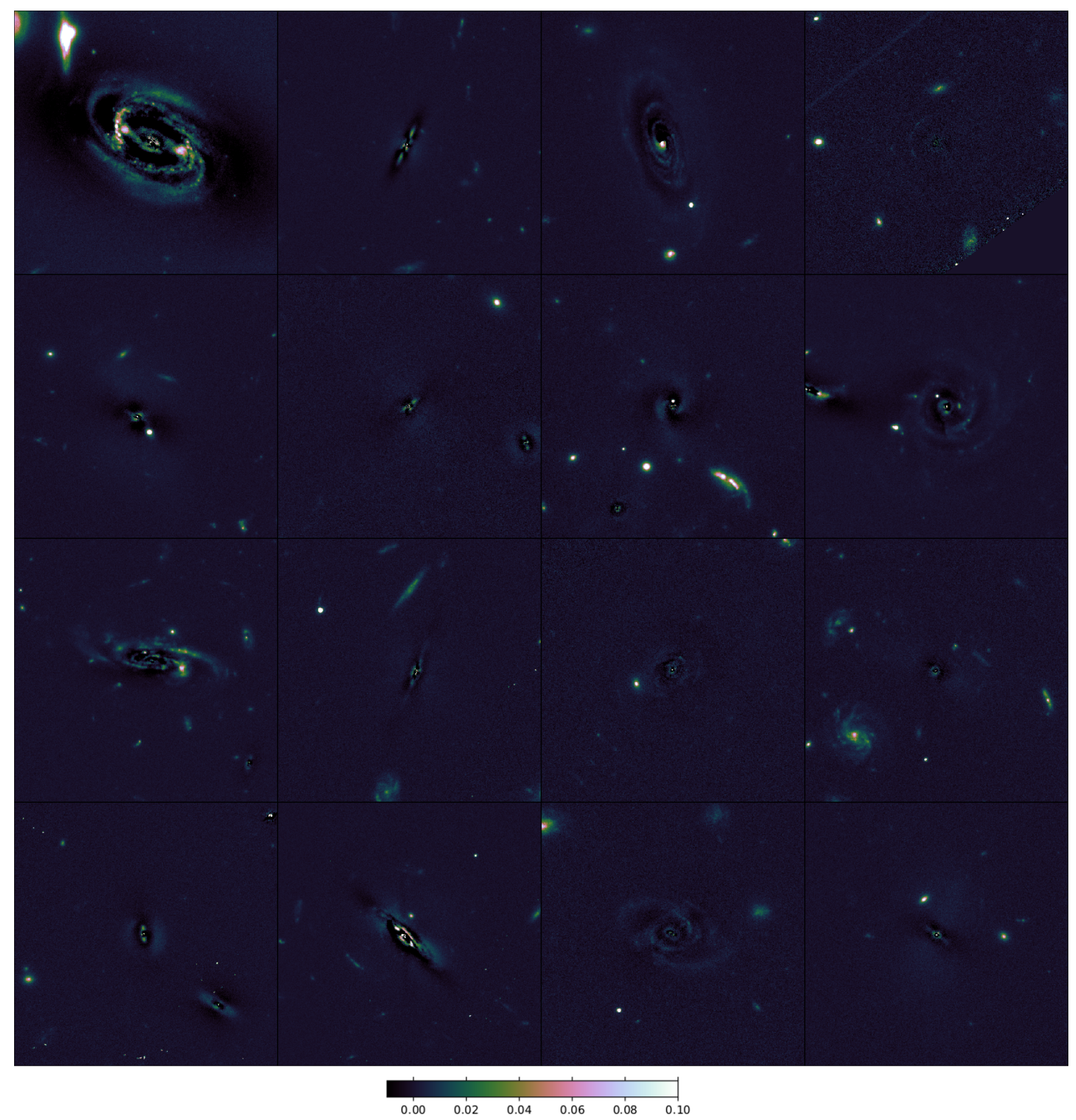

Fig. E.2. $I$-band residuals for the multi-component fits, in units of counts $\mathrm{s}^{-1}$. 